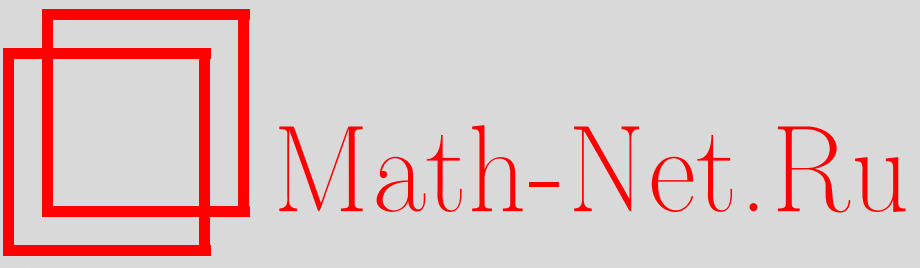

T. S. Busel, I. D. Suprunenko, The block structure of the images of regular unipotent elements from subsystem symplectic subgroups of rank 2 in irreducible representations of symplectic groups. I, Mat. Tr., 2019, Volume 22, Number 1, 68100

DOI: https://doi.org/10.33048/mattrudy.2019.22.103

Use of the all-Russian mathematical portal Math-Net.Ru implies that you have read and agreed to these terms of use

http://www . mathnet.ru/eng/agreement

Download details:

IP : 44.207 .124 .84

April 26, 2023, 12:58:01 


\title{
БЛОЧНАЯ СТРУКТУРА ОБРАЗОВ РЕГУЛЯРНЫХ УНИПОТЕНТНЫХ ЭЛЕМЕНТОВ ИЗ ПОДСИСТЕМНЫХ СИМПЛЕКТИЧЕСКИХ ПОДГРУПП РАНГА 2 В НЕПРИВОДИМЫХ ПРЕДСТАВЛЕНИЯХ СИМПЛЕКТИЧЕСКИХ ГРУПП. I
}

\author{
Т. С. Бусел, И. Д. Супруненко
}

Найдены размерности блоков Жордана образов регулярных унипотентных элементов из подсистемных подгрупп типа $C_{2}$ в $p$-ограниченных неприводимых представлениях групп типа $C_{n}$ в характеристике $p \geq 11$ с локально малыми старшими весами. Эти результаты могут быть использованы для изучения поведения унипотентных элементов в модулярных представлениях простых алгебраических групп и решения задач распознавания представлений и линейных групп.

Статья состоит из 3 -х частей. В первой части статьи содержатся предварительные леммы, необходимые для доказательства основных результатов, и исследуется случай, когда все веса ограничения рассматриваемого представления на подгруппу типа $A_{1}$, содержащую соответствующий унипотентный элемент, меньше $p$.

Ключевые слова и фразы: унипотентные элементы, размерности блоков Жордана, представления симплектических групп.

Посвящается нашему учителю и коллеге Александру Ефимовичу Залесскому с восхищением в связи с его 80-летием.

\section{§1. Введение}

В статье вычисляются размерности блоков в жордановой форме образов регулярных унипотентных элементов из подсистемных подгрупп типа $C_{2}$ в $p$-ограниченных неприводимых представлениях групп типа $C_{n}$ в характеристике $p \geq 11$ с локально малыми старшими весами. Получен аналогичный результат для нулевой характеристики. Как правило, образы таких элементов в рассматриваемых нами представлениях имеют блоки

\footnotetext{
Работа поддержана Институтом математики НАН Беларуси в рамках государственной программы «Конвергенция-2020».

(c) Т. С. Бусел, И. Д. Супруненко; 2019
} 
Жордана всех априори возможных размерностей. Существует ряд представлений, для которых это не так.

Далее $\mathbb{C}$ - поле комплексных чисел, $K$ - алгебраически замкнутое поле характеристики $p \geq 11, G=C_{n}(K), n>2, \omega_{i}, 1 \leq i \leq n,-$ фундаментальные веса группы $G, \mathbf{N}_{a}$ - множество всех целых чисел от 1 до $a$. Для элемента $z$ некоторой простой алгебраической группы и ее представления $\varphi$ символ $J_{\varphi}(z)$ обозначает множество размерностей блоков Жордана элемента $\varphi(z)$ (без учета их кратностей); аналогично определяется множество $J_{M}(z)$ для модуля $M$.

Теорема 1. Пусть $p \geq 11, G=C_{n}(K), n>2, \varphi-p$-ограниченное представление группы $G$ со старшим весом $\omega=a_{1} \omega_{1}+\cdots+a_{n} \omega_{n}, x \in G-$ регулярный унипотентный элемент из подсистемной подгруппы типа $C_{2}$. Предположим, что $3 a_{n-1}+4 a_{n}<p$. Положим $S=3 a_{1}+4\left(a_{2}+\cdots+a_{n}\right)$.

1. Пусть $S<p$. Тогда $J_{\varphi}(x)=\mathbf{N}_{S+1}$ или выполнено одно из условий:

(i) $\omega=\omega_{i}, 2 \leq i \leq n, J_{\varphi}(x)=\{1,4,5\}$;

(ii) $\omega=a_{1} \omega_{1}, a_{1}>1, J_{\varphi}(x)=\mathbf{N}_{S+1} \backslash\left\{2,3 a_{1}-4,3 a_{1}-1,3 a_{1}\right\}$;

(iii) $\omega=\omega_{1}, J_{\varphi}(x)=\{1,4\}$;

(iv) $n=3$ и ш-вес из (a)-(d):

(a) $\omega=\omega_{3}, J_{\varphi}(x)=\{4,5\}$;

(b) $\omega \in\left\{2 \omega_{1}+\omega_{3}, \omega_{1}+\omega_{3}, \omega_{1}+2 \omega_{3}, 5 \omega_{3}, \omega_{2}+\omega_{3}, \omega_{1}+\omega_{2}+\omega_{3}\right\}$,

$$
J_{\varphi}(x)=\mathbf{N}_{S+1} \backslash\{1\} ;
$$

(c) $\omega=3 \omega_{3}, J_{\varphi}(x)=\mathbf{N}_{13} \backslash\{2\}$;

(d) $\omega=2 \omega_{3}, J_{\varphi}(x)=\mathbf{N}_{9} \backslash\{1,4\}$.

2. Пусть $S \geq p$. Тогда $J_{\varphi}(x)=\mathbf{N}_{p}$ или выполнено одно из условий:

(i) $\omega=\frac{p-4}{3} \omega_{1}+\omega_{j}, 2 \leq j \leq n$,

$$
J_{\varphi}(x)=\mathbf{N}_{p} \backslash\{p-1\} ;
$$

(ii) $\omega=a_{1} \omega_{1}, a_{1}>\frac{p-1}{3}$,

$$
J_{\varphi}(x)=\left\{\begin{array}{lll}
\mathbf{N}_{p} \backslash\{2, p-2\} & \text { при } & a_{1} \neq \frac{p+1}{3}, \\
\mathbf{N}_{p} \backslash\{2, p-3, p-2\} & \text { при } & a_{1}=\frac{p+1}{3}
\end{array}\right.
$$

(iii) $\omega=\sum_{j=1}^{i} a_{j} \omega_{j}, a_{i} \geq p-3, i<n-1$,

$$
\mathbf{N}_{p} \backslash\{2, p-2\} \subset J_{\varphi}(x) \subset \mathbf{N}_{p} ;
$$

(iv) $\omega=a_{i-1} \omega_{i-1}+a_{i} \omega_{i}, a_{i-1}+a_{i}=p-1, i \leq n-1$,

$$
\mathbf{N}_{p} \backslash\{2, p-2\} \subset J_{\varphi}(x) \subset \mathbf{N}_{p} ;
$$


(v) $n=3$ и $\omega-$ вес из (a)-(e):

(a) $\omega=(p-5) \omega_{1}+\omega_{3}, J_{\varphi}(x)=\mathbf{N}_{p} \backslash\{1\}$;

(b) $\omega=4 \omega_{1}+2 \omega_{3}, p=11, J_{\varphi}(x)=\mathbf{N}_{11} \backslash\{1,10\}$;

(c) $\omega \in\left\{5 \omega_{1}+2 \omega_{3}, \omega_{1}+\omega_{2}+\omega_{3}, \omega_{1}+2 \omega_{3}\right\}, p=11, J_{\varphi}(x)=\mathbf{N}_{11} \backslash\{1\}$;

(d) $\omega=4 \omega_{1}+3 \omega_{3}, p=13, J_{\varphi}(x)=\mathbf{N}_{13} \backslash\{2\}$;

(e) $\omega=6 \omega_{1}+4 \omega_{3}, p=17, J_{\varphi}(x)=\mathbf{N}_{17} \backslash\{1\}$.

Таким образом, $\left|J_{\varphi}(x)\right| \geq p-3$ при $S \geq p$. Кроме того, $\left|J_{\varphi}(x)\right| \geq p-2$ при $\omega \neq \frac{p+1}{3} \omega_{1}$.

Теорема 2. Пусть $G_{\mathbb{C}}=C_{n}(\mathbb{C}), n>2, x_{\mathbb{C}} \in G_{\mathbb{C}}$ - регулярный унипотентный элемент из подсистемной подгруппы типа $C_{2}, \varphi-$ неприводимое представление группы $G_{\mathbb{C}}$ со старшим весом $\omega$, и пусть $\omega$ и $S$ такие, как в теореме 1. Тогда $J_{\varphi}\left(x_{\mathbb{C}}\right)$ вычисляется, как в п. 1 теоремы 1.

Доказательство теоремы 2 проводится параллельно доказательству теоремы 1 при $S<p$.

Некоторые частные случаи рассматриваемой задачи изучены А. А. Осиновской [9]. Используя обозначения теоремы 1 , предположим, что $a_{i} \neq 0$ для некоторого $i$ с $1<i<n$. Выберем максимальное такое $i$. Если при этом $a_{i}=1$, то предположим дополнительно, что $a_{j} \neq 0$ для некоторого $j$ с $1<j<i$, и выберем максимальное такое $j$. Положим $a=a_{i}$ при $a_{i}>1$ и $a=a_{i}+a_{j}$ при $a_{i}=1$. В [9] найдено множество $J_{\varphi}(x)$ в случае, когда $a_{n-1}=a_{n}=0,3 a \leq p-3$ и $a \neq 3$. Там же определены размерности блоков элемента $\varphi(x)$, не меньшие $3 a+4 a_{n}+1$, в случае, когда $3 a+4 a_{n}<p$. Для удобства читателя мы приводим доказательство теоремы 1 полностью, поскольку использование результатов [9] не привело бы к существенному сокращению текста.

При решении задачи разработаны методы получения прямых слагаемых с определенными свойствами в ограничениях неприводимых представлений групп типа $C_{n}$ на подсистемные подгруппы и некоторые подгруппы типа $A_{1}$, которые могут использоваться и для других простых алгебраических групп (см. §2). При ослаблении ограничений на характеристику поля, вероятно, в формулировке теоремы 1 было бы больше исключительных случаев.

Блочная структура образов корневых элементов в неприводимых представлениях группы типа $C_{n}(K)$ и ряда других алгебраических групп изучена М. В. Величко $[4 ; 6 ; 28]$. Для представлений с локально малыми старшими весами эта задача рассматривалась в [22; 23]. В [10] найдены размерности блоков Жордана образов регулярных унипотентных элементов из подсистемной подгруппы типа $A_{3}$ в инфинитезимально неприводимых представлениях групп типа $A_{n}$ в характеристике $\geq 11$ с локально малыми 
старшими весами (с точностью до двух размерностей для некоторых представлений специального вида). В стандартной реализации эти элементы имеют ту же нормальную форму Жордана, что и элементы, рассматриваемые в нашей статье. Естественно возникло стремление распространить результаты из [10] на представления симплектической группы. Это не происходило автоматически. Потребовалось разработать существенно новые подходы из-за различного строения систем корней специальных линейных и симплектических групп.

Детальная информация о поведении унипотентных элементов в представлениях алгебраических групп полезна для решения задач распознавания представлений и линейных групп по наличию определенных матриц. В случае положительной характеристики не приходится в ближайшем будущем ожидать решения проблемы вычисления размерностей произвольных неприводимых представлений простых алгебраических групп. В этой ситуации набор размерностей блоков Жордана (без учета их кратностей) образа фиксированного унипотентного элемента в заданном представлении - важный инвариант, который можно рассматривать как естественный ослабленный аналог нормальной формы Жордана. Результаты о свойствах образов унипотентных элементов в неприводимых модулярных представлениях полупростых алгебраических групп легко распространяются на абсолютно неприводимые представления конечных групп Шевалле в собственной характеристике, что расширяет область их потенциальных приложений.

Теорема 1 была анонсирована в [3]. К сожалению, в формулировке этой теоремы в [3] опущен п. $2(\mathrm{v})$ и неправильно указано множество $J_{\varphi}(x)$ при $\omega=\frac{p+1}{3} \omega_{1}$ в п. $2(\mathrm{ii})$.

Из-за большого объема пришлось разделить статью на 3 части. В первой части статьи содержатся предварительные леммы, необходимые для доказательства основных результатов, и доказываются часть 1 теоремы 1 и теорема 2 .

\section{§2. Обозначения и предварительные сведения}

Ниже $G=C_{n}(K), \omega_{i}$ и $\alpha_{i}, 1 \leq i \leq n,-$ фундаментальные веса и простые корни группы $G, \varepsilon_{i}, 1 \leq i \leq n,-$ веса стандартного $G$-модуля, определенные в $[2, \S 13],\langle\mu, \alpha\rangle$ - значение веса $\mu$ на корне $\alpha$ (в смысле $[12, \S 1]$ ). Символы $\omega_{i}, \varepsilon_{i}$ и $\alpha_{i}$ используются не только для группы $G$, но и для других полупростых алгебраических групп; из контекста всегда ясно, о какой группе идет речь. Нумерация простых корней алгебраических групп соответствует [1]. Напомним, что неприводимое представление полупростой алгебраической группы в характеристике $p$ называется $p$-ограниченным, 
если все коэффициенты его старшего веса меньше $p$. Если $\Gamma-$ полупростая алгебраическая группа над $\mathbb{C}$ или $K$, то $W(\Gamma)$ - ее группа Вейля, $\mathfrak{X}_{\beta}$ - корневая подгруппа, ассоциированная с корнем $\beta, \Gamma\left(\beta_{1}, \ldots, \beta_{k}\right)-$ подгруппа в $Г$, порожденная корневыми подгруппами $\mathfrak{X}_{ \pm \beta_{1}}, \ldots, \mathfrak{X}_{ \pm \beta_{k}} ; \Lambda(\Gamma)$ и $\Lambda^{+}(\Gamma)-$ множество весов и множество доминантных весов группы $Г$. Положим

$$
\mathfrak{X}_{ \pm i}=\mathfrak{X}_{ \pm \alpha_{i}}, \quad \Gamma\left(i_{1}, \ldots, i_{k}\right)=\Gamma\left(\alpha_{i_{1}}, \ldots, \alpha_{i_{k}}\right) .
$$

Используем также обозначение $\Gamma\left(i_{1}, \ldots, i_{k}, \beta\right)$ для группы $\Gamma\left(\alpha_{i_{1}}, \ldots, \alpha_{i_{k}}, \beta\right)$ и другие подобные обозначения. Ниже $X_{ \pm i, t}$ - элемент гипералгебры группы $\Gamma$, ассоциированный с корнем $\pm \alpha_{i}$ и числом $t$. Подгруппу, порожденную всеми корневыми подгруппами, ассоциированными с корнями из некоторой подсистемы системы корней группы Г, будем называть подсистемной nодгруппой. Для простой алгебраической группы Г символом $\rho_{\Gamma}$ обозначается полусумма ее положительных корней.

Далее $\operatorname{dim} M(\operatorname{dim} \varphi)$ - размерность $Г$-модуля $M$ (представления $\varphi$ ), $\Lambda(M)(\Lambda(\varphi))$ и $\Lambda^{+}(M)\left(\Lambda^{+}(\varphi)\right)$ - множества весов и доминантных весов модуля $M$ (представления $\varphi$ ). Если $\omega \in \Lambda^{+}(\Gamma)$, то $M(\omega), V(\omega)$ и $T(\omega)-$ неприводимый модуль, модуль Вейля и неразложимый тилтинг-модуль группы Г со старшим весом $\omega ; \omega(\varphi)(\omega(M))$ - старший вес представления $\varphi$ (модуля $M) ; \omega(m)$ - вес весового вектора $m$ из некоторого модуля, $M_{\lambda}$ - весовое подпространство веса $\lambda$ в модуле $M$. Напомним, что $\Gamma$-модуль называется тилтинг-модулем, если он имеет и фильтрацию модулями Вейля, и фильтрацию комодулями Вейля. Если $Г$ - простая алгебраическая группа над $K$, то $\Gamma_{\mathbb{C}}-$ простая алгебраическая группа над $\mathbb{C}$ с той же системой корней, что и Г. Для неприводимого представления $\varphi$ группы $Г$ символ $\varphi_{\mathbb{C}}$ обозначает неприводимое представление группы $\Gamma_{\mathbb{C}}$ со старшим весом $\omega(\varphi)$; аналогично определяется $\Gamma_{\mathbb{C}}$-модуль $M_{\mathbb{C}}$ для неприводимого Г-модуля $M$; при этом используется обозначение $M_{j, \mathbb{C}}$ вместо $\left(M_{j}\right)_{\mathbb{C}}$ и т. П.

Если $H$ - подгруппа в $Г$, то $M \mid H$ - ограничение Г-модуля $M$ на $H$. Предполагается, что веса и корни группы $\Gamma$ рассматриваются относительно фиксированного максимального тора $T$. Если $T \cap H-$ максимальный тор подгруппы $H$, то $\omega \mid H-$ ограничение веса $\omega$ на $T \cap H$. В этом случае для весового вектора $m$ из некоторого Г-модуля полагаем $\omega_{H}(m)=\omega(m) \mid H$. Если $M$ - неприводимый Г-модуль, то $v \in M-$ ненулевой вектор старшего веса. Символом $d_{\varphi}(x)$ обозначим степень минимального многочлена образа элемента $x \in \Gamma$ в представлении $\varphi, \operatorname{Irr} M-$ множество композиционных факторов модуля $M$ без учета их кратностей. Множество весов группы $A_{1}(K)$ отождествляется с множеством целых чисел $\mathbb{Z}: a \omega_{1} \mapsto a$. 
Рассматриваются только конечномерные представления. Далее в этом параграфе $\Gamma$ - произвольная полупростая алгебраическая группа над $\mathbb{C}$ или $K$, если не оговорено иное.

В работе существенно используются следующие факты. В силу [15, предложение 5.13] для элемента $m$ произвольного $G$-модуля, корня $\gamma$ группы $G$ и элемента $t \in K$ выполняется формула

$$
x_{\gamma}(t) m=\sum_{i=0}^{\infty} t^{i} X_{\gamma, i} m .
$$

В дальнейшем мы применяем эту формулу без специальных ссылок.

Теорема 3 [26]. Пусть $S=\Gamma\left(i_{1}, \ldots, i_{k}\right) \subseteq \Gamma, M-$ неприводимый $\Gamma-$ модуль со старшим весом $\omega, v \in M-$ ненулевой вектор старшего веса. Тогда подпространство $K S v \subseteq M$ является неприводимым $S$-модулем со старшим весом $\omega \mid S$ и прямым слагаемым $S$-модуля $M$.

Лемма 1. Пусть $1 \leq i \leq n, G_{1}=G(1, \ldots, i-1, i+1, \ldots, n)$ и $M-$ неприводимый $G_{1}$-модуль. Предположим, что $M \cong M^{*}$. Для $k \in \mathbb{Z}^{+}$положим

$$
\Omega_{k}=\left\{\lambda \in \Lambda(M) \mid \lambda=\omega-k \alpha_{i}-\sum_{j \neq i} x_{j} \alpha_{j}\right\}, \quad U_{k}=\left\langle M_{\lambda} \mid \lambda \in \Omega_{k}\right\rangle .
$$

Пусть $h=h(M)-$ максимальное число, при котором $\Omega_{h} \neq \varnothing$. Тогда $G_{1}$ модули $U_{k}$ и $U_{h-k}$ дуальны.

Доказательство. Пусть $T$ - фиксированный максимальный тор, относительно которого определены веса и корни группы $G$. Тогда $T$ нормализует подгруппу $G_{1}$. Ясно, что $U_{k}-$ прямое слагаемое ограничения $M \mid T G_{1}$. Поскольку модуль $M$ изоморфен $M^{*}$, то его младший вес равен $-\omega(M), \Omega_{h-k}=\left\{\lambda \in \Lambda(M) \mid-\lambda \in \Omega_{k}\right\}$ и $M \mid T G_{1}$ имеет прямое слагаемое $U$, изоморфное $U_{k}^{*}$. Учитывая, что $\operatorname{dim}\left(U_{k}\right)_{\lambda}=\operatorname{dim} U_{-\lambda}$ для любого веса $\lambda \in \Lambda(M)$, и анализируя действие тора $T$ на подпространствах $U_{j}$, получаем, что $U=U_{h-k}$.

Лемма 2 [20, ч. II, лемма 2.13]. Г-модуль, порожденный ненулевым вектором веса $\lambda$, неподвижным относительно всех корневых подгрупп, ассоциированных с положительными корнями, является гомоморфным образом модуля Вейля $V(\lambda)$ группы $Г$.

Лемма 3 [16]. Пусть $\Gamma=A_{2}(K), M-$ неприводимый $p$-ограниченный Г-модуль со старшим весом $\omega=a_{1} \omega_{1}+a_{2} \omega_{2}$. Тогда модуль $V(\omega)$ неприводим при $a_{1}+a_{2}<p-1$ и имеет два композиционных фактора $M(\omega)$ и $M\left(\omega-d\left(\alpha_{1}+\alpha_{2}\right)\right)$ при $a_{1}+a_{2}=p+d-2, d \geq 1$.

Лемма 4. Пусть $\Gamma=A_{2}(K), M-$ неприводимый $p$-ограниченный $\Gamma$ модуль со старшим весом $\omega=a_{1} \omega_{1}+a_{2} \omega_{2}, a_{1}+a_{2} \neq p-1, a_{2} \neq 0$, и $0<k \leq a_{1}$. Тогда кратность веса $\omega-k \alpha_{1}-\alpha_{2}$ в модуле $M$ равна 2. 
Доказательство. Положим $\lambda=\omega-k \alpha_{1}-\alpha_{2}$. Ясно, что

$$
\lambda \notin \Lambda\left(M\left(\omega-d\left(\alpha_{1}+\alpha_{2}\right)\right)\right) \text { при } d>1 \text {. }
$$

Поэтому из леммы 3 следует, что $\operatorname{dim} M_{\lambda}=\operatorname{dim} V(\omega)_{\lambda}$. В силу $[24,6.9]$ эта кратность равна 2.

Теорема 4 [11]. Пусть $\varphi-p$-ограниченное неприводимое представление группы $G$. Тогда $\Lambda(\varphi)=\Lambda\left(\varphi_{\mathbb{C}}\right)$.

Лемма $5[24,1.5]$. Пусть $M-$ неприводимый Г-модуль, $m \in M-$ ненулевой весовой вектор, $\beta$ - корень группы $\Gamma, \mathfrak{X}_{\beta}$ сохраняет $m$ и $0<$ $\langle\omega(M), \beta\rangle<p$. Тогда $X_{-\beta, c} m \neq 0$ при $0<c \leq\langle\omega(M), \beta\rangle$.

Из леммы 2.46 в [27] вытекает

Лемма 6. Пусть $M$ - неразложимый $G$-модуль со старшим весом $\sum_{i=1}^{n} a_{i} \omega_{i}$ и $v \in M-$ ненулевой вектор старшего веса. Пусть $1 \leq s, t \leq n$. Предположим, что $0<a_{t}<p$. Пусть $b_{k}=-\left\langle\alpha_{k+1}, \alpha_{k}\right\rangle$ и $c_{k}=-\left\langle\alpha_{k-1}, \alpha_{k}\right\rangle$. Для целого $d c 0<d \leq a_{t}$ определим вектор $v(s, t, d)$ следующим образом. Пусть $d_{t}=d$. Если $s>t$, то положим $d_{k}=a_{k}+d_{k-1} c_{k}$ при $s \geq k>t$. Если $s<t$, то положим $d_{k}=a_{k}+d_{k+1} b_{k}$ при $s \leq k<t$. Запишем

$$
v(s, t, d)=X_{-s, d_{s}} \ldots X_{-k, d_{k}} \ldots X_{-t, d} v \text {. }
$$

При $s=t$ положим $v(s, t, d)=X_{-s, d} v$. Тогда $v(s, t, d) \neq 0$ и $X_{m, b} v(s, t, d)=0$ для положительного $m \neq s$ и $b>0$. Следовательно, группа $\mathfrak{X}_{m}$ фиксирует $v(s, t, d)$.

Обозначение $v(s, t, d)$ многократно используется ниже.

Следствие 1. Пусть $H=G(i, i+1, \ldots, n), 1<i<n$, и $\varphi-p$-ограниченное неприводимое представление группы $G$ со старшим весом

$$
\omega(\varphi)=a_{1} \omega_{1}+\cdots+a_{n-1} \omega_{n-1}+a_{n} \omega_{n}, a_{i} \neq 0 .
$$

Предположим, что $t-$ целое число и $0<t \leq a_{i}$. Тогда $\varphi \mid H$ имеет композиционный фактор со старшим весом

$$
\left(a_{i-1}+a_{i}-t\right) \omega_{1}+\left(a_{i+1}+t\right) \omega_{2}+a_{i+2} \omega_{3}+\cdots+a_{n} \omega_{n-i+1} .
$$

Доказательство. Положим $m=v(i-1, i, t)$. В силу леммы $6 m \neq 0$ и подгруппы $\mathfrak{X}_{j}, j>i-1$, сохраняют $m$. Поэтому $m$ порождает неразложимый $H$-модуль со старшим весом $\omega_{H}(m)$. Легко видеть, что $\omega_{H}(m)$ равен указанному выше весу. Отсюда следует наше утверждение.

Предложение 1, лемма 8 и следствие 2, вероятно, известны, но мы не можем привести явные ссылки. 
Предложение 1. Пусть $U-\Gamma$-модуль. Предположим, что $\operatorname{Irr} U=$ $S_{1} \cup S_{2}, S_{i} \neq \varnothing$, и не существует неразложимых Г-модулей с двумя композиционными факторами $M_{1}$ и $M_{2}$ такими, что $M_{i} \in S_{i}, i=1,2$. Тогда $U=N_{1} \oplus N_{2}$, где $N_{i}-\Gamma$-модули и $\operatorname{Irr} N_{i}=S_{i}$.

Доказательство. Будем использовать индукцию по числу композиционных факторов модуля $U$ (здесь кратности факторов учитываются). Предположим, что $U$ имеет $l$ композиционных факторов и предложение верно для модулей с меньшим числом композиционных факторов. Очевидно, можно считать, что $l \geq 3$. Пусть $M \subset U-$ неприводимый подмодуль. Положим $\bar{U}=U / M$. Без ограничения общности будем считать, что $M \in S_{1}$. Ясно, что $S_{2} \subset \operatorname{Irr} \bar{U}$. Предположим сначала, что $\operatorname{Irr} \bar{U}=S_{2}$. Очевидно, что существует подмодуль $U_{1} \subset U$ такой, что $M \subset U_{1}$ и $U_{1}$ имеет $l-1$ композиционных факторов. Понятно, что $\operatorname{Irr}\left(U_{1} / M\right) \subset S_{2}$. Поэтому в силу предположения индукции $U_{1}=M \oplus U_{2}$, где $U_{2}-\Gamma$-модуль и $\operatorname{Irr} U_{2} \subset S_{2}$. Положим $P=U / U_{2}$. Ясно, что $P$ имеет два композиционных фактора, один из них изоморфен $M$, а другой лежит в $S_{2}$. Тогда по условию предложения $P=P_{1} \oplus P_{2}$, где $P_{1} \cong M, P_{2} \in S_{2}$. Положим $N_{1}=M$ и возьмем в качестве $N_{2}$ полный прообраз модуля $P_{2}$ в $U$. Ясно, что $\operatorname{Irr} N_{2}=S_{2}, N_{1} \cap N_{2}=0$ и модуль $N_{2}$ имеет $l-1$ композиционных факторов. Отсюда следует, что $U=N_{1} \oplus N_{2}$.

Пусть $\operatorname{Irr} \bar{U} \neq S_{2}$. В силу предположения индукции $\bar{U}=\bar{N}_{1} \oplus \bar{N}_{2}$, где $\bar{N}_{i}-\Gamma$-модули, $\operatorname{Irr} \bar{N}_{1} \subset S_{1}, \operatorname{Irr} \bar{N}_{2}=S_{2}$. Возьмем в качестве $N_{1}$ полный прообраз модуля $\bar{N}_{1}$ в $U$. Ясно, что $\operatorname{Irr} N_{1}=S_{1}$ и что для всех модулей $Q \in S_{1}$ кратности композиционного фактора, изоморфного $Q$, в модулях $N_{1}$ и $U$ совпадают. Пусть $Z-$ полный прообраз модуля $\bar{N}_{2}$ в $U$. Ввиду предположения индукции $Z=M \oplus N_{2}$, где $N_{2}-$ Г-модуль и $\operatorname{Irr} N_{2}=S_{2}$. Ясно, что для всех модулей $F \in S_{2}$ кратности композиционного фактора, изоморфного $F$, в модулях $U$ и $N_{2}$ совпадают. Так как $N_{1} \cap N_{2}=0$, отсюда следует, что $U=N_{1} \oplus N_{2}$.

Ниже будет использоваться символ $\operatorname{Ext}_{\Gamma}^{1}\left(M_{2}, M_{1}\right)$, обозначающий группу расширений $Г$-модуля $M_{1}$ с помощью $M_{2}$, т. е. множество классов эквивалентности коротких точных последовательностей Г-модулей вида

$$
0 \rightarrow M_{1} \stackrel{i}{\rightarrow} M \stackrel{j}{\rightarrow} M_{2} \rightarrow 0
$$

со стандартной операцией. Напомним, что для неприводимых модулей $M_{1}$ и $M_{2}$ модуль $M$ из такой последовательности вполне приводим при $\operatorname{Ext}_{\Gamma}^{1}\left(M_{2}, M_{1}\right)=0$.

Лемма 7 [20, ч. II, п. 2.12, формулы (1), (4)]. Пусть $M_{1}$ и $M_{2}$ - неприводимые Г-модули. Тогда

1) $\operatorname{Ext}_{\Gamma}^{1}\left(M_{1}, M_{1}\right)=0$;

2) $\operatorname{Ext}_{\Gamma}^{1}\left(M_{1}, M_{2}\right)=\operatorname{Ext}_{\Gamma}^{1}\left(M_{2}, M_{1}\right)$. 
Предложение 2 [20, ч. II, предложение 2.14]. Пусть $\lambda, \mu \in \Lambda^{+}(\Gamma)$ такие, что $\mu \ngtr \lambda$. Тогда

$$
\operatorname{Ext}_{\Gamma}^{1}(M(\lambda), M(\mu)) \cong \operatorname{Hom}_{\Gamma}\left(\operatorname{rad}_{\Gamma} V(\lambda), M(\mu)\right),
$$

где $\operatorname{rad}_{\Gamma} V(\lambda)$ - максимальный собственный подмодуль модуля $V(\lambda)$.

Лемма 8. Пусть $U-\Gamma$-модуль, $M=M(\omega)$ и $\operatorname{Irr} U=M \cup I$. Предположим, что модуль $V(\omega)$ неприводим и $M \notin \operatorname{Irr} V(\lambda)$, если $M(\lambda) \in I$. Тогда $U=N_{1} \oplus N_{2}$, где $N_{1}$ - прямая сумма нескольких копий модуля $M$, $N_{2}-\Gamma$-модуль, $\operatorname{Irr} N_{2}=I$.

Доказательство. В силу предложения 2 и леммы 7

$$
\operatorname{Ext}_{\Gamma}^{1}(M, Q)=\operatorname{Ext}_{\Gamma}^{1}(Q, M)=0 \text { для любого } Q \in I \text {. }
$$

Поэтому не существует неразложимых Г-модулей с двумя композиционными факторами, один из которых изоморфен $M$, а другой содержится в $I$. В силу предложения $1 U=N_{1} \oplus N_{2}$, где $N_{1}$ и $N_{2}-\Gamma$-модули, $\operatorname{Irr} N_{1}=M$, a $\operatorname{Irr} N_{2}=I$. Осталось доказать полную приводимость модуля $N_{1}$.

Пусть $l$ - число композиционных факторов модуля $N_{1}$. Используем индукцию по $l$. При $l=1$ наше утверждение очевидно. При $l=2$ оно следует из леммы 7 . Пусть $l>2$. Предположим, что утверждение верно для модулей, у которых меньше $l$ композиционных факторов. Очевидно, что в $N_{1}$ имеется подмодуль $S$ с $l-2$ композиционными факторами. Из леммы 7 следует, что

$$
N_{1} / S=\bar{U}_{1} \oplus \bar{U}_{2}, \text { где } \bar{U}_{i} \cong M .
$$

Пусть $U_{i}-$ полный прообраз $\bar{U}_{i}$ в $N_{1}$. Ясно, что $N_{1}=U_{1}+U_{2}$. Так как модули $U_{i}$ имеют ровно $l-1$ композиционных факторов, в силу предположения индукции каждый из них вполне приводим. Отсюда вытекает полная приводимость модуля $N_{1}$.

Следствие 2. Пусть $U-\Gamma$-модуль. Предположим, что модуль $V(\lambda)$ неприводим, если $M(\lambda) \in \operatorname{Irr} U$. Тогда модуль $U$ вполне приводим.

Доказательство. Используем индукцию по порядку $t$ множества $\operatorname{Irr} U$. При $t=1$ рассуждаем так же, как для модуля $N_{1}$ в доказательстве леммы 8. Пусть $t>1$. Предположим, что утверждение следствия верно для модулей $F$ с мощностью множества $\operatorname{Irr} F$, меньшей $t$. Зафиксируем модуль $M \in \operatorname{Irr} U$ и положим $I=\operatorname{Irr} U \backslash M$. Так как модули $V(\lambda)$ неприводимы при $M(\lambda) \in \operatorname{Irr} U$, по предложению 2 не существует неразложимых модулей с двумя композиционными факторами, один из которых изоморфен $M$, а другой содержится в $I$. Поэтому модуль $M$ и множество $I$ удовлетворяют 
условию леммы 8. В силу этой леммы $U$ есть прямая сумма нескольких копий модуля $M$ и модуля $U_{1}$ с $\operatorname{Irr} U_{1}=I$. Поскольку $|I|=t-1$, модуль $U_{1}$ вполне приводим ввиду предположения индукции. Отсюда вытекает утверждение следствия.

Следствие 3 хорошо известно, его доказательство приведено здесь, чтобы показать его связь с более общими фактами.

Следствие 3. Пусть $\Gamma=A_{1}(K), U-\Gamma$-модуль, все веса которого меньше $p$. Тогда модуль $U$ вполне приводим.

Доказательство. Известно, что модули Вейля группы Г со старшими весами, меньшими $p$, неприводимы. Поэтому наше утверждение вытекает из следствия 2.

Лемма 9 [23, лемма 10]. Пусть $\varphi$ - неприводимое представление группы $\Gamma=A_{1}(K)$ со старшим весом $a, 0 \leq a<p$. Тогда $\operatorname{dim} \varphi=a+1$ и для неединичного унипотентного элемента $z \in \Gamma$ преобразование $\varphi(z)$ имеет единственный блок Жордана размерности $a+1$.

Определение 1. Связная замкнутая подгруппа $A$ типа $A_{1}$ в простой алгебраической группе Г над $K$ называется хорошей, если образы всех корней при гомоморфизме $\sigma: \Lambda(\Gamma) \rightarrow \Lambda(A)$, индуцированном ограничением весов с максимального тора $T$ группы $\Gamma$ на максимальный тор $T_{A}=T \cap A$ в $A$, меньше $2 p-1$.

Лемма 10 [25, предложение 2.2]. Если Г - простая алгебраическая группа над $K$, то для любого элемента $x \in \Gamma$ порядка $p$ существует хорошая подгруппа $A$, содержащая $x$, и все такие подгруппы сопряжены; при этом можно выбрать систему простых корней так, что $\sigma\left(\alpha_{i}\right)$ принадлежит $\{0,1,2\}$ и совпадает с $i$-й меткой на помеченной диаграмме Дынкина элемента $x$ (здесь $\sigma-$ гомоморфизм из определения 1 ).

Известно [17, гл. 5], что для регулярного элемента $x$ число $\sigma\left(\alpha_{i}\right)$ равно 2 для всех $i$.

Лемма 11. Пусть $\Gamma$ - простая алгебраическая группа над $K, \alpha-$ максимальный короткий корень группы Г. При $\left\langle\omega+\rho_{\Gamma}, \alpha\right\rangle \leq p$ модуль Вейля $V(\omega)$ неприводим.

Доказательство. Утверждение леммы вытекает из [20, ч. II, предложение 8.19], поскольку легко заметить, что $\left\langle\omega+\rho_{\Gamma}, \beta\right\rangle \leq\left\langle\omega+\rho_{\Gamma}, \alpha\right\rangle$ для любого положительного корня $\beta$ группы $\Gamma$.

Лемма 12 [4, лемма 1]. Пусть $\lambda \in \Lambda^{+}(\Gamma)$ и модуль $V(\lambda)$ неприводим. Предположим, что $\lambda$ - максимальный вес Г-модуля $U$ и весовое подпространство этого веса одномерно в $U$. Тогда $U=V \oplus N$, где $N \cong M(\lambda)$. 
Лемма 13 [23, лемма 12]. Пусть $\Delta-$ некоторая группа, и пусть $U=$ $U_{1} \oplus \cdots \oplus U_{k}-$ прямая сумма $\Delta$-модулей. Тогда $J_{U}(z)=\bigcup_{j=1}^{k} J_{U_{j}}(z)$ для элемента $z \in \Delta$.

Следствие 4 [5, следствие 8]. Пусть $H-$ подгруппа группы $\Delta$ и $z \in H$. Предположим, что для $\Delta$-модуля $U$ ограничение $U \mid H$ вполне приводимо. Тогда $J_{M}(z) \subset J_{U}(z)$ для любого композиционного фактора $M$ модуля $U$.

В теореме $5 J_{k}$ - блок Жордана размерности $k$ с единицей на диагонали, $J(h)$ - множество размерностей блоков (без учета кратностей) в форме Жордана элемента $h$ из соответствующей линейной группы.

Теорема 5 [14, гл. VIII, теорема 2.7]. Пусть $1 \leq s \leq t \leq p$. Тогда

$$
J\left(J_{s} \otimes J_{t}\right)=\left\{\begin{array}{lll}
\{t-s+1, t-s+3, \ldots, t+s-1\} & \text { при } & s+t \leq p ; \\
\{p, t-s+1, \ldots, 2 p-t-s-1\} & \text { при } & s+t>p .
\end{array}\right.
$$

$B$ частности, $J\left(J_{s} \otimes J_{p}\right)=\{p\}$.

Лемма 14 [25, лемма 1.1].

(a) Для каждого доминантного веса $\lambda$ группы Г существует неразложимый тилтинг-модуль $T(\lambda)$ со старшим весом $\lambda$, определенный однозначно с точностью до изоморфизма.

(b) Любой тилтинг-модуль есть прямая сумма неразложимых тилтингмодулей вида $T(\lambda)$.

(c) Прямое слагаемое тилтинг-модуля также является тилтинг-модулем.

(d) Тензорное произведение тилтинг-модулей - тилтинг-модуль.

В лемме 15, предложении 3 и следствии 5 рассматриваются тилтингмодули группы $A_{1}(K)$.

Лемма 15 [25, леммы 1.2, 1.3]. При $0 \leq c<p$

$$
T(c) \cong V(c) \cong M(c) .
$$

При $p \leq c \leq 2 p-2$ положим $c=r+p$. Тогда максимальный подмодуль $M$ модуля $V(c)$ изоморфен $M(p-r-2)$ и $V(c) / M \cong M(c)$. В модуле $T(c)$ имеется фильтрация

$$
\begin{gathered}
T(c)=M_{1} \supset M_{2} \supset M_{3} \supset M_{4}=0 \\
\text { c } M_{1} / M_{2} \cong M_{3} \cong M(p-r-2) \text { и } M_{2} / M_{3} \cong M(r+p) ; \\
\operatorname{dim}(T(c))=2 p .
\end{gathered}
$$

В этом случае модуль $T(c)$ проективен для группы $A_{1}(p)$, а значит, нетривиальный унипотентный элемент имеет в этом модуле два блока размерности $р$. 
Предложение 3 [18, пример 2]. Пусть $m \geq p$. Тогда

$$
T(m) \cong T(p-1+r) \otimes T(s)^{F r},
$$

где $r$ и $s$ определяются из равенства

$$
m+1-p=r+p s, \quad 0 \leq r \leq p-1,
$$

$F r$ - морфизм Фробениуса группы $A_{1}(K)$, задаваемый возведением элементов поля $K$ в степень $p$.

Следствие 5. При $m \geq p$ нетривиальные унипотентные элементы имеют в модуле $T(m)$ только блоки размерности $p$.

Лемма 16. Пусть $A \subset \Gamma-$ связная полупростая замкнутая подгруппа, $\omega \in \Lambda^{+}(\Gamma)$. Предположим, что модуль Вейля $V(\omega)$ неприводим и что существуют веса $\lambda_{1}$ и $\lambda_{2} \in \Lambda^{+}(\Gamma)$ такие, что $\omega=\lambda_{1}+\lambda_{2}$ и ограничения $M\left(\lambda_{1}\right) \mid A$ и $M\left(\lambda_{2}\right) \mid A$ - тилтинг-модули. Тогда $M(\omega) \mid A$ - тилтингмодуль.

Доказательство. Ясно, что размерность весового пространства веса $\omega$ в модуле $M\left(\lambda_{1}\right) \otimes M\left(\lambda_{2}\right)$ равна 1 . Тогда согласно лемме $12 M(\omega)$ - прямое слагаемое этого тензорного произведения. Остается использовать утверждения (c) и (d) леммы 14.

Следствие 6. Пусть $Г-$ простая алгебраическая группа, $A \subset \Gamma-$ связная полупростая замкнутая подгруппа, $M-$ неприводимый Г-модуль со старшим весом $\omega$. Предположим, что ограничения на $A$ неприводимых Г-модулей с фундаментальными старшими весами - тилтинг-модули и что $\left\langle\omega+\rho_{\Gamma}, \alpha\right\rangle \leq p$ для максимального короткого корня $\alpha$ группы Г. Тогда $M \mid A-$ тилтинг-модуль.

Доказательство. Пусть $r-$ ранг группы $\Gamma, \omega=\sum_{i=1}^{r} a_{i} \omega_{i}$. Положим $s=\sum_{i=1}^{r} a_{i}$. Используем индукцию по $s$. При $s=1$ утверждение следствия верно по условию. Пусть $s>1$ и $a_{j} \neq 0$ для некоторого $j$. Положим $\lambda_{1}=\omega-\omega_{j}, \lambda_{2}=\omega_{j}$. В силу предположения индукции и условия следствия $M\left(\lambda_{i}\right) \mid A$ - тилтинг-модуль при $i=1,2$. Модуль $V(\omega)$ неприводим ввиду леммы 11. Теперь наше утверждение следует из леммы 16.

Следствие 7. Пусть $\Gamma=A_{n}(K), A$ - образ вполне приводимого представления группы $A_{1}(K)$ с р-ограниченными неприводимыми компонентами, $M-$ неприводимый Г-модуль со старшим весом $a_{1} \omega_{1}$ и $a_{1}<p$. Тогда $M \mid A-$ тилтинг-модуль.

Доказательство. Ясно, что $M\left(\omega_{1}\right) \mid A$ - тилтинг-модуль. Известно, что $G$-модуль $V\left(a_{1} \omega_{1}\right)$ неприводим. Достаточно несколько раз применить лемму 16. 
Лемма 17. Пусть $H \subset G-$ подсистемная подгруппа типа $C_{2}$ и $a<p$. Тогда ограничение $M\left(a \omega_{1}\right) \mid H$ вполне приводимо и

$$
\operatorname{Irr}\left(M\left(a \omega_{1}\right) \mid H\right)=\left\{M\left(k \omega_{1}\right) \mid 0 \leq k \leq a\right\} .
$$

Доказательство. Утверждение следует из [8, предложение 2.8].

Теорема 6 [24, 8.1]. Неприводимые модули группы $G$ со старшими весами аш при $a<p$ или $a \omega_{i}+(p-1-a) \omega_{i+1}$, где $1 \leq i<n$ и $a \neq 0$ для $i=n-1$, эквивалентны ограничениям на $G$ неприводимых модулей группы $A_{2 n-1}(K)$ с такими же старшими весами.

Предложение 4 [8, предложение 1.2]. Пусть $\Delta=A_{r}(K), V$ - естественный $\Delta$-модуль, $k$ и $j$ - целые неотрицательные числа, $j<p$. При $r=k(p-1)+j \leq(n+1)(p-1) r$-я усеченная симметрическая степень $S^{r, p}(V)$ является неприводимым модулем со старшим весом

$$
(p-1-j) \omega_{k}+j \omega_{k+1} .
$$

Bсе весовые подпространства этого модуля одномерны. В частности, $r$-я симметрическая степень $S^{r}(V)$ - неприводимый $\Delta$-модуль со старшим весом $r \omega_{1}$ при $r<p$.

Лемма 18. Пусть $\Delta=A_{r}(K), k+t=r-1, H_{1}=A_{k}(K), H_{2}=A_{t}(K)$, $V_{j}$ - естественный модуль группы $H_{j}, j=1,2, V$ - естественный $\Delta$ модуль, $H \cong H_{1} H_{2}$ - подсистемная подгруппа в $\Delta, S^{i, p}(V)-i$-я усеченная симметрическая степень модуля $V$. Тогда

$$
S^{i, p} V \cong \bigoplus_{\substack{i_{1}+i_{2}=i, 0 \leq i_{1} \leq(k+1)(p-1), 0 \leq i_{2} \leq(t+1)(p-1)}} S^{i_{1}, p} V_{1} \otimes S^{i_{2}, p} V_{2} .
$$

Для доказательства действие подгруппы $H$ рассматривается в стандартных базисах соответствующих модулей, построенных по фиксированным базисам модулей $V_{1}$ и $V_{2}$.

$\mathrm{B}$ теоремах 7 и 8 обозначения $\varepsilon_{i}, 1 \leq i \leq r$, используются для весов естественного модуля произвольной алгебраической группы типа $C_{r}$, а не только для группы $G$. Из контекста ясно, о какой группе идет речь.

Теорема 7. Пусть $\Gamma=C_{r}(K), r \geq 2, x \in \Gamma-$ регулярный унипотентный элемент из подсистемной подгруппы типа $C_{2}, A-$ хорошая $A_{1}$ подгруппа, содержащая $x, \sigma: \Lambda(\Gamma) \rightarrow \Lambda(A)-$ гомоморфизм из леммы 10 . Тогда $\sigma\left(\varepsilon_{1}\right)=3, \sigma\left(\varepsilon_{2}\right)=1, \sigma\left(\varepsilon_{i}\right)=0$ при $3 \leq i \leq r$.

Пусть $\varphi$ - неприводимое $p$-ограниченное представление группы $Г$ со старшим весом $\sum_{i=1}^{r} a_{i} \omega_{i}$. Тогда

$$
\begin{gathered}
\max _{\mu \in \Lambda(\varphi)}\{\sigma(\mu)\}=3 a_{1}+4\left(a_{2}+\cdots+a_{r}\right), \\
d_{\varphi}(x)=\min \left\{p, 1+3 a_{1}+4\left(a_{2}+\cdots+a_{r}\right)\right\} .
\end{gathered}
$$


Доказательство. Пусть $x_{\mathbb{C}} \in \Gamma_{\mathbb{C}}-$ элемент с той же нормальной формой Жордана в естественном модуле, что и $x$.

Нетрудно установить, что $x_{\mathbb{C}}($ и $x$ ) в естественном модуле имеет один блок Жордана размерности 4 и блоки размерности 1. Положим

$$
N(x)=(3,1,0, \ldots, 0,-1,-3) \quad(\text { в } N(x) 2 r \text { чисел }) .
$$

В силу [13, предложение 2.12] набор

$$
\left(\sigma\left(\varepsilon_{1}\right), \sigma\left(-\varepsilon_{1}\right), \ldots, \sigma\left(\varepsilon_{r}\right), \sigma\left(-\varepsilon_{r}\right)\right)
$$

совпадает с набором $N(x)$ с учетом кратностей. Так как $\sigma\left(\alpha_{i}\right) \geq 0$, отсюда следуют формулы для $\sigma\left(\varepsilon_{i}\right)$ и ясно, что

$$
\max _{\mu \in \Lambda(\varphi)}\{\sigma(\mu)\}=\sigma(\omega(\varphi)) \text {. }
$$

Поскольку $\omega_{i}=\sum_{j=1}^{i} \varepsilon_{j}$, получаем

$$
\sigma(\omega(\varphi))=3 a_{1}+4\left(a_{2}+\cdots+a_{r}\right) .
$$

Согласно [13, теорема 1.1]

$$
d_{\varphi}(x)=\min \left\{p, d_{\varphi_{\mathbb{C}}}\left(x_{\mathbb{C}}\right)\right\} .
$$

Пусть $\tau_{j}$ - неприводимое представление группы $\Gamma_{\mathbb{C}}$ со старшим весом $\omega_{j}, 1 \leq j \leq r, m_{j}=d_{\tau_{j}}\left(x_{\mathbb{C}}\right)-1$. Ввиду [13, алгоритм 1.4] число $m_{j}$ равно сумме $j$ максимальных чисел из набора $N(x)$. Поэтому

$$
m_{1}=3, \quad m_{2}=\cdots=m_{r}=4 .
$$

На основании [13, предложение 1.3] заключаем, что

$$
d_{\varphi_{\mathbb{C}}}\left(x_{\mathbb{C}}\right)=1+\sum_{i=1}^{r} a_{i} m_{i}=1+3 a_{1}+4\left(a_{2}+\cdots+a_{r}\right) .
$$

Положим

$$
Q_{n}=\left\{\left(a_{1}, \ldots, a_{n}\right) \mid a_{1} \geq a_{2} \geq \cdots \geq a_{n}, a_{i} \in \mathbb{N}\right\} .
$$

Если

$$
a=\left(a_{1}, a_{2}, \ldots, a_{n}\right) \in Q_{n} \quad \text { и } b=\left(b_{1}, b_{2}, \ldots, b_{n-1}\right) \in Q_{n-1},
$$

то $b<a$ означает, что

$$
a_{1} \geq b_{1} \geq a_{2} \geq \cdots \geq b_{n-1} \geq a_{n} .
$$

В следующей теореме символом $V_{a}$ обозначим неприводимый $C_{n}(\mathbb{C})$-модуль со старшим весом $a_{1} \varepsilon_{1}+\cdots+a_{n} \varepsilon_{n}$, где $a \in Q_{n}$. 
Теорема 8 [7, теорема 11]. Пусть $H \subset C_{n}(\mathbb{C})$ - подсистемная подгруппа типа $C_{n-1}$. Тогда

$$
V_{a} \mid H \cong \bigoplus_{b<(a, 0)} \bigoplus_{c<b} V_{c}, \quad \text { где } a, b \in Q_{n}, \quad c \in Q_{n-1} .
$$

Предложение 5. Пусть $V_{a}-$ неприводимый модуль для группы $C_{3}(\mathbb{C})$ со старшим весом $a_{1} \omega_{1}+a_{2} \omega_{2}+a_{3} \omega_{3}, H \subset C_{3}(\mathbb{C})-$ подсистемная подгруппа типа $C_{2}$. Тогда множество композиционных факторов ограничения $V_{a} \mid H$ состоит из всех неприводимых модулей со старшими весами вида $d_{1} \omega_{1}+d_{2} \omega_{2}$ с $a_{3} \leq d_{1}+d_{2} \leq a_{1}+a_{2}+a_{3}$ и $d_{2} \leq a_{2}+a_{3}$.

Доказательство. Воспользуемся теоремой 8. В обозначениях этой теоремы $a$ равно $\left(a_{1}+a_{2}+a_{3}, a_{2}+a_{3}, a_{3}\right), b$ пробегает множество наборов $\left(b_{1}, b_{2}, b_{3}\right)$ таких, что

$$
a_{2}+a_{3} \leq b_{1} \leq a_{1}+a_{2}+a_{3}, a_{3} \leq b_{2} \leq a_{2}+a_{3}, \quad 0 \leq b_{3} \leq a_{3} .
$$

Имеем

$$
V_{a} \mid H \cong \bigoplus_{b<(a, 0)} \bigoplus_{c<b} V_{c}
$$

Поэтому $b_{2} \leq c_{1} \leq b_{1}, b_{3} \leq c_{2} \leq b_{2}$ для всех слагаемых $V_{c}$. Для пары целых неотрицательных чисел $d=\left(d_{1}, d_{2}\right)$ пусть $M(d)$ - неприводимый $H$-модуль со старшим весом $d_{1} \omega_{1}+d_{2} \omega_{2}$,

$$
\mathcal{D}=\left\{d \mid M(d) \text { - композиционный фактор модуля } V_{a} \mid H\right\} .
$$

Поскольку $M(d) \cong V_{c}$ при $c=\left(d_{1}+d_{2}, d_{2}\right)$, отсюда следует, что $d_{1}$ и $d_{2}$ удовлетворяют соотношениям $a_{3} \leq d_{1}+d_{2} \leq a_{1}+a_{2}+a_{3}$ и $d_{2} \leq a_{2}+a_{3}$ при $d \in \mathcal{D}$.

С другой стороны, пусть $d_{1}, d_{2}-$ неотрицательные целые числа,

$$
a_{3} \leq d_{1}+d_{2} \leq a_{1}+a_{2}+a_{3} \text { и } d_{2} \leq a_{2}+a_{3} .
$$

Положим

$$
c_{1}=d_{1}+d_{2}, \quad c_{2}=d_{2}, \quad b_{1}=\max \left\{c_{1}, a_{2}+a_{3}\right\}, \quad b_{2}=\max \left\{a_{3}, c_{2}\right\}, \quad b_{3}=0 .
$$

Тогда $c=\left(d_{1}+d_{2}, d_{2}\right)<b=\left(b_{1}, b_{2}, b_{3}\right)$ и по теореме $8 V_{c} \cong M(d)-$ прямое слагаемое ограничения $V_{a} \mid H$.

Лемма 19. Пусть $\Gamma=C_{r}(K), r \geq 2, x \in \Gamma-$ регулярный унипотентный элемент из подсистемной подгруппы типа $C_{2}, M-$ неприводимый $\Gamma$ модуль со старшим весом $\omega=a_{1} \omega_{1}+\cdots+a_{r} \omega_{r}$. Предположим, что модуль Вейля $V(\omega)$ неприводим и $3 a_{1}+4\left(a_{2}+\cdots+a_{r}\right)<p$. Тогда размерности блоков Жордана элемента $x$ в модуле $M$ такие же, как у аналогичного элемента группы $\Gamma_{\mathbb{C}}$ в модуле $M_{\mathbb{C}}$. 
Доказательство. Пусть $x_{\mathbb{C}} \in \Gamma_{\mathbb{C}}-$ регулярный унипотентный элемент из подсистемной подгруппы типа $C_{2}, A$ и $\sigma$ - подгруппа типа $A_{1}$ и гомоморфизм, описанные в теореме 7. Легко видеть, что нормальные формы Жордана элементов $x$ и $x_{\mathbb{C}}$ в естественных модулях групп $\Gamma$ и $\Gamma_{\mathbb{C}}$ совпадают. В силу предложения 2.2 и предшествующего ему замечания из [25] в $\Gamma_{\mathbb{C}}$ существует замкнутая в топологии Зарисского подгруппа $A_{\mathbb{C}} \cong A_{1}(\mathbb{C})$ такая, что $x_{\mathbb{C}} \in A_{\mathbb{C}}, T_{\mathbb{C}} \cap A_{\mathbb{C}}-$ максимальный тор в $A_{\mathbb{C}}$ для некоторого максимального тора $T_{\mathbb{C}} \subset \Gamma_{\mathbb{C}}$ и ограничение весов с $T_{\mathbb{C}}$ на $T_{\mathbb{C}} \cap A_{\mathbb{C}}$ задает гомоморфизм $\sigma$.

Так как $M \cong V(\omega)$, из сказанного выше следует, что кратности весов модулей $M_{\mathbb{C}} \mid A_{\mathbb{C}}$ и $M \mid A$ совпадают. Ввиду теоремы $7 \sigma(\mu)<p$ для любого веса $\mu \in \Lambda(M)$. Тогда по следствию $3 M \mid A-$ прямая сумма $p$-ограниченных неприводимых модулей. Таким образом, размерности блоков Жордана элементов $x_{\mathbb{C}}$ и $x$ в модулях $M_{\mathbb{C}}$ и $M$ соответственно однозначно определяются кратностями весов модулей $M_{\mathbb{C}} \mid A_{\mathbb{C}}$ и $M \mid A$ и поэтому совпадают.

Теорема 9 [21, теорема 6 и табл. 2]. Пусть $\varphi-$ неприводимое представление группы $C_{2}(\mathbb{C})$ со старшим весом $m_{1} \omega_{1}+m_{2} \omega_{2}$. Тогда образ регулярного унипотентного элемента в представлении $\varphi$ имеет блоки всех размерностей

$$
i \equiv 3 m_{1}+4 m_{2}+1 \quad(\bmod 2), \quad \text { где } 1 \leq i \leq 3 m_{1}+4 m_{2}+1,
$$

за исключением весов и блоков, указанных в табл. 1. В частности, при $m_{1}>10$ и $m_{2}>4$ имеются блоки всех априори возможных размерностей.

Далее если $\Gamma=C_{r}(K)$, то $z \in \Gamma-$ регулярный унипотентный элемент из подсистемной подгруппы типа $C_{2}, z_{\mathbb{C}}-$ аналогичный элемент в группе $\Gamma_{\mathbb{C}}, A_{\Gamma} \subset \Gamma$ и $A_{\Gamma, \mathbb{C}} \subset \Gamma_{\mathbb{C}}-$ подгруппы типа $A_{1}$, содержащие элементы $z$ и $z_{\mathbb{C}}$ и описанные в теореме 7 и доказательстве леммы 19 соответственно.

Теорема 10. Пусть

$$
\Gamma=C_{3}(K), \quad \Gamma_{1}=\Gamma(2,3),
$$

$\omega$ - доминантный вес, $M=M(\omega)$,

$$
\Delta=\left\{\lambda \in \Lambda\left(\Gamma_{1}\right)\left|M(\lambda) \in \operatorname{Irr} M_{\mathbb{C}}\right| \Gamma_{1, \mathbb{C}}\right\},
$$

$z$ - регулярный унипотентный элемент группы $\Gamma_{1}, \alpha$ - максимальный короткий корень группы Г. Предположим, что

$$
\left\langle\omega+\rho_{\Gamma}, \alpha\right\rangle \leq p, \quad \delta \in \Delta, \quad N=M(\delta)-\text { неприводимый } \Gamma_{1} \text {-модуль. }
$$

Тогда $J_{N}(z) \subset J_{M}(z)$. 
Таблица 1

\begin{tabular}{|c|c|c|}
\hline$m_{1}$ & $m_{2}$ & размер отсутствующего \\
блока \\
\hline $1(\bmod 2)>1$ & 0 & $3 m_{1}+4 m_{2}-1,2$ \\
1 & $0,2(\bmod 3)$ & 2 \\
1 & $1(\bmod 3)$ & 4 \\
3 & $3(\bmod 6)$ & 2 \\
2 & 0 & $3 m_{1}+4 m_{2}-1=5,1$ \\
$2(\bmod 4)>2$ & 0 & $3 m_{1}+4 m_{2}-1,5,1$ \\
$0(\bmod 4)$ & 0 & $3 m_{1}+4 m_{2}-1,3$ \\
0 & 1 & $3 m_{1}+4 m_{2}-1=3,1$ \\
0 & 2 & $3 m_{1}+4 m_{2}-1=7,3,1$ \\
0 & 3 & $3 m_{1}+4 m_{2}-1=11,5,3$ \\
0 & $0(\bmod 3)>3$ & $3 m_{1}+4 m_{2}-1,11,5,3$ \\
2 & $>0$ & $3 m_{1}+4 m_{2}-1,7,3,1$ \\
2 & 1 & 1 \\
$0(\bmod 3)>2$ & 1 \\
$2(\bmod 4)>2$ & 2 & 1 \\
6 & 1,2 & 1 \\
4,10 & $1,4(\bmod 6)>1$ & 4 \\
$2,6,10(\bmod 12)$ и $>6$ & 4 & \\
\hline
\end{tabular}

Доказательство. В силу леммы 11 модуль Вейля $V(\omega)$ неприводим. Покажем, что модуль $V(\lambda)$ неприводим, если $M(\lambda) \in \operatorname{Irr} M \mid \Gamma_{1}$. Пусть $\beta$ - максимальный короткий корень группы $\Gamma_{1}$. Так как $\lambda \in \Lambda^{+}(\Gamma)$ и $\left\langle\rho_{\Gamma}, \alpha\right\rangle=5$, то $\langle\mu, \alpha\rangle \leq\langle\omega, \alpha\rangle \leq p-5$ для любого $\mu \in \Lambda(M)$. Поскольку $\lambda=\nu \mid \Gamma_{1}$ для некоторого $\nu \in \Lambda(M)$ и корни $\alpha$ и $\beta$ лежат в одной $W$ орбите, заключаем, что $\langle\lambda, \alpha\rangle \leq p-5$ и $\left\langle\lambda+\rho_{\Gamma_{1}}, \beta\right\rangle \leq p-2$. Поэтому модуль $V(\lambda)$ неприводим. Поскольку формальные характеры модулей $M$ и $M_{\mathbb{C}}$ совпадают, получаем, что $\operatorname{Irr} M\left|\Gamma_{1}=\operatorname{Irr} M_{\mathbb{C}}\right| \Gamma_{1, \mathbb{C}}$. Тогда ввиду леммы $11 M \mid \Gamma_{1}$ - прямая сумма неприводимых модулей. Из сказанного выше следует, что при $\delta \in \Delta$ в ограничении $M$ на $\Gamma_{1}$ имеется прямое слагаемое $N \cong M(\delta)$. Поэтому $J_{N}(z) \subset J_{M}(z)$.

Теорема 11. Пусть $\Gamma=C_{2}(K), \omega=a_{1} \omega_{1}+a_{2} \omega_{2} \in \Lambda^{+}(\Gamma), a_{1}+2 a_{2} \leq$ $p-3$. Тогда модуль Вейля $V(\omega)$ неприводим и ограничение $V(\omega) \mid A_{\Gamma}-$ тилтинг-модуль. В частности, это верно, если $3 a_{1}+4 a_{2} \leq p+5$.

Доказательство. Сначала докажем неприводимость модуля Вейля. Пусть $\alpha$ - максимальный короткий корень группы Г. Легко видеть, что

$$
\alpha=\alpha_{1}+\alpha_{2} \text { и }\left\langle\omega+\rho_{\Gamma}, \alpha\right\rangle=a_{1}+2 a_{2}+3 \leq p .
$$

Согласно лемме 11 модуль Вейля $V(\omega)$ неприводим. 
Пусть $\lambda-$ фундаментальный вес и $N=V(\lambda)$. Из теоремы 7 следует, что $\max _{\mu \in \Lambda(N)} \sigma(\mu) \leq 4$ для гомоморфизма $\sigma$ из этой теоремы, поэтому в силу следствия $3 \mathrm{~N} \mid A_{\Gamma}$ - прямая сумма неприводимых $p$-ограниченных модулей, изоморфных модулям Вейля с соответствующими старшими весами. Далее используем следствие 6 и заключаем, что $V(\omega) \mid A_{\Gamma}$ - тилтингмодуль.

Покажем, что $a_{1}+2 a_{2} \leq p-3$ при $3 a_{1}+4 a_{2} \leq p+5$. Действительно, если при этом $2 a_{1}+2 a_{2} \geq 8$, то $a_{1}+2 a_{2}+3 \leq 3 a_{1}+4 a_{2}-5 \leq p$. Если же $2 a_{1}+2 a_{2}<8$, то $a_{1}+a_{2} \leq 3$ и $a_{1}+2 a_{2} \leq 6<p-3$.

Лемма 20. Пусть $\Gamma=C_{2}(K), \omega=a_{1} \omega_{1}+a_{2} \omega_{2}, M=M(\omega)-$ неприводимый Г-модуль. Предположим, что $a_{1}+2 a_{2} \leq p-3,3 a_{1}+4 a_{2}<2 p$, $a<p-1$ и $M(a) \notin \operatorname{Irr} N$ для любой неразложимой компоненты $N$ модуля $M \mid A_{\Gamma}$ такой, что $N \cong T(b) c b \geq p$. В этом случае $a+1 \in J_{M}(z)$ в точности тогда, когда $a+1 \in J_{M_{\mathbb{C}}}\left(z_{\mathbb{C}}\right)$. В частности, это верно, если $3 a_{1}+4 a_{2}=p+x$ и $а<p-x-2$.

Доказательство. По теореме 11 модуль $V(\omega)$ неприводим и $M \mid A_{\Gamma}-$ тилтинг-модуль. Поэтому $\operatorname{dim} M_{\lambda}=\operatorname{dim}\left(M_{\mathbb{C}}\right)_{\lambda}$ для любого веса $\lambda \in \Lambda(M)$ и факторы фильтрации ограничений $M \mid A_{\Gamma}$ и $M_{\mathbb{C}} \mid A_{\Gamma, \mathbb{C}}$ модулями Вейля совпадают (с учетом кратностей). Ясно, что если $V(a)$ встречается в такой фильтрации для модуля $M_{\mathbb{C}} \mid A_{\Gamma, \mathbb{C}}$, то $V(a)$ является прямым слагаемым этого ограничения. Из условия леммы следует, что если $V(a)$ встречается среди факторов фильтрации модуля $M \mid A_{\Gamma}$ модулями Вейля, то $V(a) \cong M(a)$ - прямое слагаемое ограничения $M \mid \Gamma$, так как фактор $V(a)$ не может встретиться в фильтрации неразложимых компонент $T(b)$ с $b \geq p$. Отсюда вытекает, что модули $M_{\mathbb{C}} \mid A_{\Gamma, \mathbb{C}}$ и $M \mid A_{\Gamma}$ одновременно имеют или не имеют неразложимую компоненту, изоморфную $M(a)$. Поскольку $J_{N}(z)=\{p\}$ при $N \cong T(b)$ с $b \geq p$, получаем первое утверждение леммы. Из теоремы 7 следует, что $b \leq 3 a_{1}+4 a_{2}$ для любой неразложимой компоненты $T(b)$ модуля $M \mid A_{\Gamma}$. В силу леммы 15 $M(b) \notin \operatorname{Irr} T(p+q)$, если $0 \leq q<p-1$ и $b<p-q-2$. Отсюда вытекает второе утверждение леммы.

Далее $G_{1}=G(n-1, n), \gamma$ и $\beta$ - максимальные короткие корни групп $G$ и $G_{1}$ соответственно.

Предложение 6. Пусть $\Gamma=G(i, \ldots, n), i \leq n-2, x-$ регулярный унипотентный элемент из подгруппы $G_{1}, A \subset G_{1}$ и $\sigma: \Lambda\left(G_{1}\right) \rightarrow \mathbb{Z}-A_{1}$ подгруппа, содержащая $x$, и гомоморфизм из теоремы $7, N-\Gamma$-модуль c единственным максимальным весом $\lambda, s=\max _{\mu \in \Lambda\left(N \mid G_{1}\right)} \sigma(\mu)$. Положим $b_{j}=\left\langle\lambda, \alpha_{j}\right\rangle$ при $i \leq j \leq n$. Предположим, что $b_{i}+2\left(b_{i+1}+\cdots+b_{n}\right) \leq p-3$. Тогда модуль $N \mid G_{1}$ вполне приводим, $J_{N}(x)=\bigcup_{F \in \operatorname{Irr}\left(N \mid G_{1}\right)} J_{F}(x)$ и $F \mid A-$ 
тилтинг-модуль при $F \in \operatorname{Irr}\left(N \mid G_{1}\right), s=3 b_{i}+4\left(b_{i+1}+\cdots+b_{n}\right)$. В частности, утверждение предложения справедливо, если $s \leq p+5$.

Доказательство. Пусть $\delta$ - максимальный короткий корень группы $Г$. Нетрудно заметить, что для любого веса $\mu \in \Lambda(N)$

$$
\langle\mu, \delta\rangle \leq\langle\lambda, \delta\rangle=b_{i}+2\left(b_{i+1}+\cdots+b_{n}\right) \leq p-3 .
$$

Поскольку $\beta$ и $\delta$ лежат в одной $W$-орбите, очевидно, что $\langle\mu, \beta\rangle \leq p-3$ для любого веса $\mu \in \Lambda(N)$. По лемме 11 модуль $V(\tau)$ неприводим, если $M(\tau) \in \operatorname{Irr} N \mid G_{1}$. В силу следствия 2 модуль $N \mid G_{1}$ вполне приводим. Отсюда вытекает утверждение предложения о $J_{N}(x)$. Ввиду следствия 6 $F \mid A-$ тилтинг-модуль, если $F \in \operatorname{Irr}\left(N \mid G_{1}\right)$.

Пусть $\sigma_{\Gamma}$ - гомоморфизм из теоремы 7 , связанный с подгруппой $A_{\Gamma}$. Так как подгруппы $A_{\Gamma}$ и $A$ сопряжены в $\Gamma$, легко видеть, что

$$
s=\max _{\mu \in \Lambda(N)} \sigma_{\Gamma}(N)
$$

Теперь из доказательства теоремы 7 следует, что $s=3 b_{i}+4\left(b_{i+1}+\cdots+b_{n}\right)$. Пусть $s \leq p+5$. Рассуждая, как в доказательстве теоремы 11, заключаем, что $b_{i}+2\left(b_{i+1}+\cdots+b_{n}\right) \leq p-3$. Отсюда вытекает последнее утверждение предложения.

Предложение 7. Пусть $M-C_{2}(\mathbb{C})$-модуль со старшим весом $\omega=$ $a \omega_{1}+b \omega_{2}$. Тогда размерности указанных ниже весовых подпространств определяются следующими таблицами.

\begin{tabular}{|r|r|c|}
\hline$a$ & $b$ & $\operatorname{dim} M_{\omega-2 \alpha_{1}-\alpha_{2}}$ \\
\hline$>1$ & $>0$ & 3 \\
1 & $>0$ & 2 \\
$>1$ & 0 & 2 \\
1 & 0 & 1 \\
0 & $>0$ & 1 \\
\hline
\end{tabular}

\begin{tabular}{|r|r|c|}
\hline$a$ & $b$ & $\operatorname{dim} M_{\omega-\alpha_{1}-2 \alpha_{2}}$ \\
\hline$>0$ & $>1$ & 2 \\
$>0$ & 1 & 1 \\
0 & $>1$ & 1 \\
0 & 1 & 0 \\
$>0$ & 0 & 0 \\
\hline
\end{tabular}

\begin{tabular}{|r|r|c|}
\hline$a$ & $b$ & $\operatorname{dim} M_{\omega-3 \alpha_{1}-\alpha_{2}}$ \\
\hline$>2$ & $>0$ & 3 \\
2 & $>0$ & 2 \\
1 & $>0$ & 1 \\
$>2$ & 0 & 2 \\
2 & 0 & 1 \\
1 & 0 & 0 \\
0 & $>0$ & 0 \\
\hline
\end{tabular}

\begin{tabular}{|r|r|c|}
\hline$a$ & $b$ & $\operatorname{dim} M_{\omega-2 \alpha_{1}-2 \alpha_{2}}$ \\
\hline$>1$ & $>1$ & 4 \\
$>1$ & 1 & 3 \\
$>1$ & 0 & 1 \\
1 & $>1$ & 3 \\
1 & 1 & 2 \\
1 & 0 & 0 \\
0 & $>1$ & 2 \\
0 & 1 & 0 \\
\hline
\end{tabular}




\begin{tabular}{|r|r|c|}
\hline$a$ & $b$ & $\operatorname{dim} M_{\omega-\alpha_{1}-3 \alpha_{2}}$ \\
\hline$>0$ & $>2$ & 2 \\
$>0$ & 2 & 1 \\
$>0$ & 1 & 0 \\
$>0$ & 0 & 0 \\
0 & $>2$ & 1 \\
0 & $\leq 2$ & 0 \\
\hline
\end{tabular}

Доказательство. Пусть $d_{\lambda}-$ кратность веса $\lambda$ модуля $M, W-$ группа Вейля группы типа $C_{2}, s_{i} \in W$ - отражения, ассоциированные с корнями $\alpha_{i}$. Положим $\rho=\rho_{C_{2}(\mathbb{C})}$. Используем формулу Вейля

$$
d_{\lambda}=-\sum_{w \in W, w \neq 1} \varepsilon(w) d_{\lambda+\rho-w(\rho)}
$$

где $\varepsilon(w)=(-1)^{l(w)}, l(w)$ - длина элемента $w$ относительно семейства отражений $s_{i}$. Известно, что $|W|=8$ и $W$ - полупрямое произведение группы $S_{2}$, действующей перестановками на множестве $\left\{\varepsilon_{1}, \varepsilon_{2}\right\}$, и группы порядка 4 , переводящей веса $\varepsilon_{i}$ в $\pm \varepsilon_{i}$. Легко видеть, что

$$
\begin{aligned}
& s_{1}\left(\varepsilon_{1}\right)=\varepsilon_{2}, \quad s_{1}\left(\varepsilon_{2}\right)=\varepsilon_{1}, \quad s_{1}(\rho)=2 \varepsilon_{2}+\varepsilon_{1} ; \\
& s_{2}\left(\varepsilon_{1}\right)=\varepsilon_{1}, \quad s_{2}\left(\varepsilon_{2}\right)=-\varepsilon_{2}, \quad s_{2}(\rho)=2 \varepsilon_{1}-\varepsilon_{2} \text {; } \\
& s_{1} s_{2}\left(\varepsilon_{1}\right)=\varepsilon_{2}, \quad s_{1} s_{2}\left(\varepsilon_{2}\right)=-\varepsilon_{1}, \quad s_{1} s_{2}(\rho)=2 \varepsilon_{2}-\varepsilon_{1} ; \quad\left(s_{1} s_{2}\right)^{2}=-1 .
\end{aligned}
$$

Заметим, что $W=\left\{ \pm 1, \pm s_{1}, \pm s_{2}, \pm s_{1} s_{2}\right\}$. Теперь нетрудно показать, чTо

$$
\begin{aligned}
d_{\lambda}= & d_{\lambda+\alpha_{1}}+d_{\lambda+\alpha_{2}}-d_{\lambda+3 \alpha_{1}+\alpha_{2}} \\
& -d_{\lambda+\alpha_{1}+2 \alpha_{2}}-d_{\lambda+4 \alpha_{1}+3 \alpha_{2}}+d_{\lambda+4 \alpha_{1}+2 \alpha_{2}}+d_{\lambda+3 \alpha_{1}+3 \alpha_{2}} .
\end{aligned}
$$

Для завершения доказательства применим формулу (3) к весам из таблиц.

Далее $M-$ неприводимый $G$-модуль со старшим весом $\omega=a_{1} \omega_{1}+\cdots+$ $a_{n} \omega_{n}, x \in G_{1}-$ регулярный унипотентный элемент, $A=A_{G_{1}}$. Для формулировки лемм 21 и 22 нам понадобятся дополнительные обозначения. Предположим, что $a_{1}>0$. Пусть $b \in \mathbb{N}$ и $b \leq a_{1}$. Обозначим символом $\Lambda_{b, 1}$ подмножество в $\Lambda(M)$, состоящее из всех весов вида $\omega-b \alpha_{1}-\sum_{j=2}^{n} k_{j} \alpha_{j}$, и положим $N_{b, 1}=\left\langle M_{\lambda} \mid \lambda \in \Lambda_{b, 1}\right\rangle$.

Пусть $a_{2}>0$ (теперь мы не предполагаем, что $a_{1} \neq 0$ ), $c \in \mathbb{N}$ и $c \leq a_{2}$. Обозначим символом $\Lambda_{c, 2}$ совокупность всех весов из $\Lambda(M)$ вида

$$
\omega-\left(a_{1}+c\right) \alpha_{1}-\sum_{j=2}^{n} k_{j} \alpha_{j}
$$

и положим $N_{c, 2}=\left\langle M_{\lambda} \mid \lambda \in \Lambda_{c, 2}\right\rangle$. 
Пусть $\Gamma=G(2, \ldots, n)$. Легко видеть, что подпространства $N_{b, 1}$ и $N_{c, 2}$ при подходящих $b$ и $c$ являются Г-модулями и прямыми слагаемыми модуля $M \mid \Gamma$.

Лемма 21. Пусть $a_{1}>0, b \in \mathbb{N}$ и $b \leq a_{1}$. Предположим, что

$$
\left(a_{2}+b\right)+2 \sum_{j=3}^{n} a_{j} \leq p-3 .
$$

Тогда $N_{b, 1} \mid G_{1}$ - вполне приводимый модуль, для любого композиционного фактора $F$ ограничения $N_{b, 1} \mid G_{1}$ множество $J_{F}(x)$ содержится в $J_{M}(x)$ и $F \mid A$ - тилтинг-модуль. В частности, утверждение леммы справедливо, если

$$
3\left(a_{2}+b\right)+4 \sum_{j=3}^{n} a_{j} \leq p+5 .
$$

Доказательство. В силу леммы $6 \mu=\omega-b \alpha_{1} \in \Lambda(M)$. Ясно, что $\mu \mid \Gamma$ - максимальный вес модуля $N_{b, 1}$ и что $\left\langle\mu, \alpha_{2}\right\rangle=a_{2}+b$ и $\left\langle\mu, \alpha_{i}\right\rangle=a_{i}$ при $3 \leq i \leq n$. Остается воспользоваться предложением 6 и теоремой 3 (при $b=0)$.

Лемма 22. Пусть $a_{2}>0, c \in \mathbb{N}$ и $0<c \leq a_{2}$. Предположим, что

$$
c+\left(a_{1}+a_{2}\right)+2 \sum_{j=3}^{n} a_{j} \leq p-3 .
$$

Тогда $N_{c, 2} \mid G_{1}$ - вполне приводимый модуль, для любого композиционного фактора $F$ ограничения $N_{c, 2} \mid G_{1}$ множество $J_{F}(x)$ содержится в $J_{M}(x)$ и $F \mid A-$ тилтинг-модуль. В частности, утверждение леммы справедливо, если

$$
c+3\left(a_{1}+a_{2}\right)+4 \sum_{j=3}^{n} a_{j} \leq p+5 .
$$

Доказательство. Положим $m=v(1, c, 2), \mu=\omega(m)$. В силу леммы 6 $m \neq 0$. Ясно, что $m \in N_{c, 2}$. Заметим, что

$$
\nu=\omega-\left(a_{1}+c\right) \alpha_{1}-d \alpha_{2}-\sum_{j=3}^{n} k_{j} \alpha_{j} \notin \Lambda(M) \text { при } d<c .
$$

Действительно,

$$
\left\langle\nu, \alpha_{1}\right\rangle=d+a_{1}-2\left(a_{1}+c\right)=d-c-a_{1}-c<-\left(a_{1}+c\right) .
$$


Пусть $s_{1} \in W$ - отражение, ассоциированное с корнем $\alpha_{1}$. Тогда

$$
s_{1}(\nu)=\nu+\left(a_{1}+c+c-d\right) \alpha_{1} \notin \Lambda(M),
$$

и поэтому $\nu \notin \Lambda(M)$, а значит, $\mu \mid \Gamma$ - максимальный вес модуля $N_{c, 2}$.

Легко видеть, что

$$
\left\langle\mu, \alpha_{2}\right\rangle=a_{1}+a_{2}-c, \quad\left\langle\mu, \alpha_{3}\right\rangle=a_{3}+c, \quad\left\langle\mu, \alpha_{i}\right\rangle=a_{i} \text { при } i \geq 4 .
$$

Для завершения доказательства используем предложение 6.

Следствие 8. В условиях лемм 21 и 22 для любого композиционного фактора $U$ Г-модуля $N_{b, 1}$ или $N_{c, 2}$ множество $J_{U}(x)$ содержится в $J_{M}(x)$.

Доказательство непосредственно вытекает из лемм 21, 22 и следствия 4.

Очевидно, что следствие 8 дает новую информацию лишь при $n>3$.

Далее $\mathbf{N}_{a}^{o}$ и $\mathbf{N}_{a}^{e}-$ множества нечетных и четных натуральных чисел из $\mathbf{N}_{a}$ соответственно.

\section{§3. Представления с малыми старшими весами}

Доказательства теорем 1 и 2 основаны на построении набора прямых слагаемых с определенными свойствами в ограничениях рассматриваемого модуля на подсистемные подгруппы типа $C_{2}$ и подгруппу типа $A_{1}$, содержащую рассматриваемый элемент. Эти слагаемые оказываются тилтингмодулями с не слишком большими относительно характеристики старшими весами. Существенно используются результаты А. А. Осиновской $[21$, теорема 6] о блочной структуре образов регулярных унипотентных элементов в неприводимых представлениях группы $C_{2}(\mathbb{C})$ и информация о строении тилтинг-модулей группы типа $A_{1}$ с весами, не превосходящими $2 p-2$ (лемма 15).

Далее $\rho=\rho_{G}, \varphi$ - неприводимое представление со старшим весом $\omega(\varphi)=a_{1} \omega_{1}+\cdots+a_{n} \omega_{n}$, удовлетворяющим условиям теоремы $1 ; M-$ неприводимый $G$-модуль, где реализуется представление $\varphi ; x \in G_{1}-$ регулярный унипотентный элемент, $A \subset G_{1}$ и $\sigma-A_{1}$-подгруппа, содержащая $x$, и гомоморфизм из леммы $10, G_{1, \mathbb{C}}=G_{\mathbb{C}}(n-1, n), x_{\mathbb{C}} \in G_{1, \mathbb{C}}-$ регулярный унипотентный элемент. Положим

$$
\mathcal{S}(\omega)=\left\{\mu \mid M(\mu) \in \operatorname{Irr}\left(M_{\mathbb{C}} \mid G_{1, \mathbb{C}}\right)\right\} .
$$

Вспомним, что регулярные унипотентные элементы произвольных подсистемных подгрупп типа $C_{2}$ в $G$ сопряжены с $x$. При доказательстве теоремы 1 мы фактически показываем, что $\varphi(y)$ имеет блок определенного размера для такого элемента $y$.

В этом параграфе доказывается теорема 1 в случае, когда

$$
S=3 a_{1}+4 a_{2}+\cdots+4 a_{n}<p .
$$


Лемма 23. Пусть $S<p$. Тогда модуль $M \mid A$ вполне приводим и для любого композиционного фактора $F$ ограничения $M \mid G_{1}$ модуль Вейля $V(\omega(F))$ неприводим и $J_{F}(x)=J_{F_{\mathbb{C}}}\left(x_{\mathbb{C}}\right)$.

Доказательство. В силу теоремы 7 и следствия $3 M \mid A-$ вполне приводимый модуль с $p$-ограниченными неприводимыми компонентами.

Пусть $F-$ композиционный фактор ограничения $M \mid G_{1}$ со старшим весом $\lambda=b_{1} \omega_{1}+b_{2} \omega_{2}$. Согласно теореме 7

$$
3 b_{1}+4 b_{2} \in \Lambda(F \mid A) \subset \Lambda(M \mid A),
$$

и поэтому $3 b_{1}+4 b_{2} \leq S<p$. Из леммы 19 и теоремы 11 следует, что модуль $V(\lambda)$ неприводим и $J_{F}(x)=J_{F_{\mathbb{C}}}\left(x_{\mathbb{C}}\right)$.

В §3 будем использовать следствие 4 без дополнительных пояснений. В силу теоремы 7 имеем $J_{M}(x) \subset \mathbf{N}_{S+1}$.

3.1. $\omega=a_{1} \omega_{1}$. Здесь рассматривается случай, когда $\omega=a_{1} \omega_{1}, a_{1}<\frac{p}{3}$. По лемме 17

$$
\operatorname{Irr}\left(M \mid G_{1}\right)=\left\{M\left(k \omega_{1}\right) \mid 0 \leq k \leq a_{1}\right\} .
$$

Положим $M\left(k \omega_{1}\right)=F_{k}$. Ввиду теоремы 6 и леммы $19 J_{F}(x)=J_{F_{\mathbb{C}}}\left(x_{\mathbb{C}}\right)$ при $F=F_{k}$. Поэтому из теоремы 9 вытекает следующее:

(a) $2,3 a_{1}-4,3 a_{1}-1,3 a_{1} \notin J_{M}(x)$;

(b) $\mathbf{N}_{S+1} \backslash\left\{2,3,5,3 a_{1}-4,3 a_{1}-1,3 a_{1}\right\} \subset J_{F_{0}}(x) \cup J_{F_{a_{1}-1}}(x) \cup J_{F_{a_{1}}}(x)$.

Поясним, что

$$
d_{F_{k}}(x)<\left\{\begin{array}{lll}
3 a_{1}-4 & \text { при } k<a_{1}-1 ; \\
3 a_{1}-1 & \text { при } k<a_{1} .
\end{array}\right.
$$

Поэтому $3 a_{1}-4 \notin J_{M}(x)$, так как $3 a_{1}-4 \notin J_{F_{a_{1}-1}}(x) \cup J_{F_{a_{1}}}(x)$, и $3 a_{1}-1,3 a_{1} \notin$ $J_{M}(x)$, ибо $3 a_{1}-1,3 a_{1} \notin J_{F_{a_{1}}}(x)$.

В силу теоремы 9 имеем $3 \in J_{F_{2}}(x)$ и $5 \in J_{F_{4}}(x)$. Поэтому $3 \in J_{M}(x)$ при $a_{1} \geq 2$ и $5 \in J_{M}(x)$ при $a_{1} \geq 4$. Отсюда следуют утверждения 1(ii) и 1 (iii) теоремы 1.

3.2. $n=3$. Здесь предполагается, что $n=3$. Известно, что $\gamma=\alpha_{1}+$ $2 \alpha_{2}+\alpha_{3}$. Легко проверить, что

$$
\langle\omega+\rho, \gamma\rangle=a_{1}+2 a_{2}+2 a_{3}+5
$$

Если $a_{1}+a_{2}+a_{3} \geq 3$, то

$$
a_{1}+2 a_{2}+2 a_{3} \leq 3 a_{1}+4 a_{2}+4 a_{3}-6 \text { и }\langle\omega+\rho, \gamma\rangle<p .
$$


При $a_{1}+a_{2}+a_{3} \leq 2$ выполняется $\langle\omega+\rho, \gamma\rangle \leq 9<p$. Поэтому в силу леммы 11 модуль $V(\omega)$ неприводим. Тогда $J_{M}(x)=J_{M_{\mathbb{C}}}\left(x_{\mathbb{C}}\right)$ ввиду леммы 19 . Отсюда следует, что

$$
J_{F}\left(x_{\mathbb{C}}\right) \subset J_{M}(x)
$$

для любого композиционного фактора $F$ ограничения $M_{\mathbb{C}} \mid G_{1, \mathbb{C}}$. В этом пункте формула (4) применяется без дополнительных комментариев. Для поиска нужного фактора $F$ используется предложение 5.

В силу доказанного в п. 3.1 можно считать, что $a_{2}+a_{3}>0$.

Положим

$$
\mu_{1}=a_{1} \omega_{1}+\left(a_{2}+a_{3}\right) \omega_{2}, \quad \mu_{2}=\left(a_{1}+1\right) \omega_{1}+\left(a_{2}+a_{3}-1\right) \omega_{2} .
$$

При $a_{1}>0$ введем также вес $\mu_{3}=\left(a_{1}-1\right) \omega_{1}+\left(a_{2}+a_{3}\right) \omega_{2}$. Пусть $M_{i}=M\left(\mu_{i}\right)$ - неприводимый $C_{2}(\mathbb{C})$-модуль, $1 \leq i \leq 3$. Из предложения 5 следует, что $M_{1}, M_{2} \in \operatorname{Irr}\left(M_{\mathbb{C}} \mid G_{1, \mathbb{C}}\right)$ и что $M_{3} \in \operatorname{Irr}\left(M_{\mathbb{C}} \mid G_{1, \mathbb{C}}\right)$ при $a_{1}>0$.

Положим $\mathcal{J}_{1}=J_{M_{1}}\left(x_{\mathbb{C}}\right), \mathcal{J}_{2}=J_{M_{2}}\left(x_{\mathbb{C}}\right), J=\mathcal{J}_{1} \cup \mathcal{J}_{2}$,

$$
\begin{aligned}
& I_{1}=\left\{a \mid a \equiv a_{1}+1 \quad(\bmod 2), a \leq S+1\right\}, \\
& I_{2}=\left\{a \mid a \equiv a_{1}(\bmod 2), a \leq S\right\} .
\end{aligned}
$$

Если $\mu_{1}$ и $\mu_{2}$ оба не являются исключительными весами из табл. 1, то в силу теоремы $9 J_{M_{1}}\left(x_{\mathbb{C}}\right)=I_{1}, J_{M_{2}}\left(x_{\mathbb{C}}\right)=I_{2}$. Тогда $J_{M}(x)=\mathbf{N}_{S+1}$.

Теперь рассмотрим все случаи, когда хотя бы один из весов $\mu_{1}$ или $\mu_{2}-$ исключительный вес из табл. 1 . Положим $b_{1}=a_{1}, b_{2}=a_{2}+a_{3}, c_{1}=a_{1}+1$ и $c_{2}=a_{2}+a_{3}-1$. Тогда

$$
\mu_{1}=b_{1} \omega_{1}+b_{2} \omega_{2}, \quad \mu_{2}=c_{1} \omega_{1}+c_{2} \omega_{2} .
$$

Заметим, что $b_{2} \neq 0$ и $c_{1} \neq 0$.

В пп. 3.2.1-3.2.6 все утверждения о том, что какой-либо вес лежит в $\mathcal{S}(\omega)$, следуют из предложения 5 .

3.2.1. $b_{2}=1$. В этом пункте $\omega=a_{1} \omega_{1}+\omega_{2}$ или $a_{1} \omega_{1}+\omega_{3}$. Очевидно, что в этой ситуации $c_{2}=0$. В силу предложения 5 имеем

$$
\begin{array}{ll}
\mathcal{S}\left(\omega_{2}\right)=\left\{0, \omega_{1}, \omega_{2}\right\}, & \mathcal{S}\left(\omega_{1}+\omega_{2}\right)=\left\{0, \omega_{1}, \omega_{2}, 2 \omega_{1}, \omega_{1}+\omega_{2}\right\}, \\
\mathcal{S}\left(\omega_{3}\right)=\left\{\omega_{1}, \omega_{2}\right\}, & \mathcal{S}\left(\omega_{1}+\omega_{3}\right)=\left\{\omega_{1}, \omega_{2}, 2 \omega_{1}, \omega_{1}+\omega_{2}\right\} .
\end{array}
$$

Поэтому из теоремы 9 следует, что

$$
J_{M}(x)=\left\{\begin{array}{lll}
\{1,4,5\} & \text { при } \omega=\omega_{2} ; \\
\{4,5\} & \text { при } \omega=\omega_{3} ; \\
\mathbf{N}_{8} & \text { при } \omega=\omega_{1}+\omega_{2} ; \\
\mathbf{N}_{8} \backslash\{1\} & \text { при } \omega=\omega_{1}+\omega_{3} .
\end{array}\right.
$$


Пусть $a_{1}>1$. Из теоремы 9 вытекает:

(i) $J=\mathbf{N}_{S+1} \backslash\{1,2, S-2\}$ при $a_{1} \equiv 0(\bmod 2)$;

(ii) $J=\mathbf{N}_{S+1} \backslash\{1,5, S-2\}$ при $a_{1} \equiv 1(\bmod 4)$;

(iii) $J=\mathbf{N}_{S+1} \backslash\{3, S-2\} \quad$ при $a_{1} \equiv 3(\bmod 4)$.

В силу теоремы $7 d_{M_{3}}\left(x_{\mathbb{C}}\right)=S-2$. Поскольку $M_{3} \in \mathcal{S}(\omega)$ при $a_{1}>1$, отсюда получаем, что

$$
S-2 \in J_{M_{3}}\left(x_{\mathbb{C}}\right) \subset J_{M}(x) .
$$

Справедливо следующее:

1) $0 \in \mathcal{S}(\omega)$ при $\omega=a_{1} \omega_{1}+\omega_{2}$;

2) $4 \omega_{1} \in \mathcal{S}(\omega)$ при $\omega=a_{1} \omega_{1}+\omega_{3}$ и $a_{1} \geq 3$;

3) $\omega_{2}, 2 \omega_{1}, \omega_{1}+\omega_{2} \in \mathcal{S}(\omega)$ во всех рассматриваемых случаях;

4) $\mathcal{S}\left(2 \omega_{1}+\omega_{3}\right)=\left\{\omega_{1}, \omega_{2}, \omega_{1}+\omega_{2}, 2 \omega_{1}, 2 \omega_{1}+\omega_{2}, 3 \omega_{1}\right\}$.

Тогда в силу теоремы 9

$$
J_{M}(x)= \begin{cases}\mathbf{N}_{S+1} & \text { при } \omega \neq 2 \omega_{1}+\omega_{3} ; \\ \mathbf{N}_{S+1} \backslash\{1\} & \text { при } \omega=2 \omega_{1}+\omega_{3} .\end{cases}
$$

Далее считаем, что $b_{2}>1$.

3.2.2. $b_{1}=0$. В этом пункте $\omega=a_{2} \omega_{2}+a_{3} \omega_{3}$. Ясно, что $c_{1}=1$. Используя теорему 9 , получаем

$$
\mathbf{N}_{S+1} \backslash\{1,2,3,4,5,7,11, S-1\} \subset J \subset J_{M}(x) .
$$

Заметим, что $\lambda=2 \omega_{1}+\left(a_{2}+a_{3}-2\right) \omega_{2} \in \mathcal{S}(\omega)$.

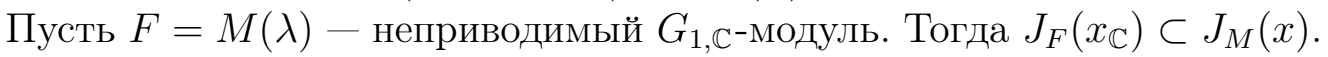
Из теоремы 9 следует, что

$$
\begin{aligned}
& \mathbf{N}_{S+1}^{o} \backslash\{1\} \subset J_{M}(x) \text { при } a_{2}+a_{3}>2, \\
& \mathbf{N}_{9}^{o} \backslash\{1,5\} \subset J_{M}(x) \text { при } a_{2}+a_{3}=2 \text {, } \\
& 5 \in \mathcal{J}_{1} \text { при } a_{2}+a_{3}=2 \text {. }
\end{aligned}
$$

Таким образом, доказано, что во всех ситуациях $\mathbf{N}_{S+1}^{o} \backslash\{1\} \subset J_{M}(x)$.

Положим

$$
\mu=\left\{\begin{array}{lll}
8 \omega_{1}+\left(a_{2}+a_{3}-8\right) \omega_{2} & \text { при } a_{2}+a_{3}>9 ; \\
\left(a_{2}+a_{3}\right) \omega_{2} & \text { при } a_{2}+a_{3} \in\{3,6,9\} ; \\
8 \omega_{1} & \text { при } a_{2}+a_{3}=8 ; \\
4 \omega_{1}+\left(a_{2}+a_{3}-4\right) \omega_{2} & \text { при } a_{2}+a_{3}=4 \text { или } 7 ; \\
4 \omega_{1} & \text { при } a_{2}+a_{3}=5, a_{2} \neq 0 ; \\
0 & \text { при } \omega=2 \omega_{2} .
\end{array}\right.
$$




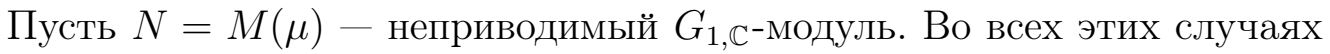
$\mu \in \mathcal{S}(\omega)$ и по теореме 9 число 1 принадлежит $J_{N}\left(x_{\mathbb{C}}\right) \subset J_{M}(x)$. Имеем

$$
\begin{aligned}
\mathcal{S}\left(\omega_{2}+\omega_{3}\right) & =\left\{2 \omega_{1}, \omega_{1}+\omega_{2}, 2 \omega_{2}, \omega_{1}, \omega_{2}\right\}, \\
\mathcal{S}\left(3 \omega_{3}\right) & =\left\{3 \omega_{1}, 2 \omega_{1}+\omega_{2}, \omega_{1}+2 \omega_{2}, 3 \omega_{2}\right\}, \\
\mathcal{S}\left(2 \omega_{3}\right) & =\left\{2 \omega_{1}, \omega_{1}+\omega_{2}, 2 \omega_{2}\right\} \\
\mathcal{S}\left(5 \omega_{3}\right) & =\left\{5 \omega_{1}, 4 \omega_{1}+\omega_{2}, 3 \omega_{1}+2 \omega_{2}, 2 \omega_{1}+3 \omega_{2}, \omega_{1}+4 \omega_{2}, 5 \omega_{2}\right\} .
\end{aligned}
$$

В силу теоремы 7

$$
J_{M}(x)=\left\{\begin{array}{lll}
\mathbf{N}_{S+1} \backslash\{1\} & \text { при } \omega=\omega_{2}+\omega_{3} \text { или } 5 \omega_{3} ; \\
\mathbf{N}_{9} \backslash\{1,4\} & \text { при } \omega=2 \omega_{3} ; \\
\mathbf{N}_{13} \backslash\{2\} & \text { при } \omega=3 \omega_{3} .
\end{array}\right.
$$

Остается выяснить, когда 2 и 4 принадлежат $J_{M}(x)$. Можно считать, что $\omega \notin\left\{\omega_{2}+\omega_{3}, 3 \omega_{3}, 2 \omega_{3}, 5 \omega_{3}\right\}$, так как эти случаи уже рассмотрены.

Положим

$$
\nu= \begin{cases}5 \omega_{1}+\left(a_{2}+a_{3}-5\right) \omega_{2} & \text { при } a_{2}+a_{3}>5 ; \\ 3 \omega_{1}+2 \omega_{2} & \text { при } a_{2}+a_{3}=5 ; \\ 3 \omega_{1}+\omega_{2} & \text { при } a_{2}+a_{3}=4 ; \\ \omega_{1}+\omega_{2} & \text { при } a_{2}+a_{3}=3, a_{2} \neq 0 ; \\ \omega_{1} & \text { при } \omega=2 \omega_{2} .\end{cases}
$$

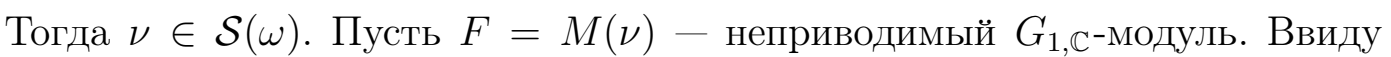
теоремы 9

$$
\begin{aligned}
\{2,4\} & \subset J_{F}\left(x_{\mathbb{C}}\right) \text { при } a_{2}+a_{3}>3, \\
2 \in J_{F}\left(x_{\mathbb{C}}\right) & \text { при } \quad a_{2}+a_{3}=3, a_{2} \neq 0, \\
4 \in J_{F}\left(x_{\mathbb{C}}\right) & \text { при } \omega=2 \omega_{2} .
\end{aligned}
$$

Легко видеть, что

$$
\mu_{2}=\left\{\begin{array}{lll}
\omega_{1}+2 \omega_{2} & \text { при } & a_{2}+a_{3}=3 \\
\omega_{1}+\omega_{2} & \text { при } & \omega=2 \omega_{2} .
\end{array}\right.
$$

Теорема 9 влечет, что $4 \in \mathcal{J}_{2}$ в первом случае и $2 \in \mathcal{J}_{2}$ во втором случае. Отсюда следует, что $\{2,4\} \subset J_{M}(x)$ для всех весов, рассматриваемых в этом абзаце. Итак, при $b_{1}=0$ справедлива теорема 1.

Далее предполагаем, что $b_{1}>0$.

3.2.3. $b_{1}=1$. В этом пункте $\omega=\omega_{1}+a_{2} \omega_{2}+a_{3} \omega_{3}$. Ясно, что $c_{1}=2$. В силу теоремы 9 заключаем, что

$$
\begin{aligned}
& \mathcal{J}_{1}=I_{1} \backslash\{2\} \quad \text { при } a_{2}+a_{3} \equiv 0,2 \quad(\bmod 3), \\
& \mathcal{J}_{1}=I_{1} \backslash\{4\} \quad \text { при } a_{2}+a_{3} \equiv 1 \quad(\bmod 3), \\
& \mathcal{J}_{2}=I_{2} \backslash\{1\} .
\end{aligned}
$$


Положим

$$
\begin{aligned}
& \mu_{1}=\left\{\begin{array}{lll}
8 \omega_{1}+\left(a_{2}+a_{3}-7\right) \omega_{2} & \text { при } a_{2}+a_{3} \geq 9 ; \\
8 \omega_{1} & \text { при } a_{2}+a_{3}=7 \text { или } 8 ; \\
4 \omega_{1}+\left(a_{2}+a_{3}-3\right) \omega_{2} & \text { при } a_{2}+a_{3}=3,5 \text { или } 6 ; \\
4 \omega_{1} & \text { при } a_{2}+a_{3}=4 ; \\
0 & \text { при } \omega=\omega_{1}+2 \omega_{2} ;
\end{array}\right. \\
& \mu_{2}=\left\{\begin{array}{lll}
5 \omega_{1}+\left(a_{2}+a_{3}-4\right) \omega_{2} & \text { при } a_{2}+a_{3} \geq 5 ; \\
3 \omega_{1}+\omega_{2} & \text { при } a_{2}+a_{3}=3 \text { или } 4 ; \\
\omega_{1}+\omega_{2} & \text { при } \omega=\omega_{1}+2 \omega_{2} .
\end{array}\right.
\end{aligned}
$$

Во всех случаях $\mu_{1}, \mu_{2} \in \mathcal{S}(\omega)$. Пусть $F_{i}=M\left(\mu_{i}\right)-$ неприводимые $C_{1, \mathbb{C}^{-}}$ модули, $i=1,2$. Из теоремы 9 следует, что

$$
\begin{aligned}
& 1 \in J_{F_{1}}\left(x_{\mathbb{C}}\right) \subset J_{M}(x),\{2,4\} \subset J_{F_{2}}\left(x_{\mathbb{C}}\right) \subset J_{M}(x) \text { при } a_{2}+a_{3}>2, \\
& 2 \in J_{F_{2}}\left(x_{\mathbb{C}}\right) \subset J_{M}(x) \text { при } \omega=\omega_{1}+2 \omega_{2} .
\end{aligned}
$$

Значит, во всех этих случаях $J_{M}(x)=\mathbf{N}_{S+1}$.

\section{Имеем}

$\mathcal{S}(\omega)=\left\{\omega_{1}+2 \omega_{2}, 2 \omega_{1}+\omega_{2}, 3 \omega_{1}, 2 \omega_{2}, \omega_{1}+\omega_{2}, 2 \omega_{1}, \omega_{2}, \omega_{1}\right\}$ при $\omega=\omega_{1}+\omega_{2}+\omega_{3} ;$ $\mathcal{S}(\omega)=\left\{2 \omega_{1}+\omega_{2}, \omega_{1}+2 \omega_{2}, 3 \omega_{1}, \omega_{1}+\omega_{2}, 2 \omega_{1}, 2 \omega_{2},\right\}$ при $\omega=\omega_{1}+2 \omega_{3}$.

Ввиду теоремы $9 J_{M}(x)=\mathbf{N}_{12} \backslash\{1\}$ для этих весов.

Далее считаем, что $b_{1}>1$.

3.2.4. $b_{1}=2$. В этом пункте $\omega=2 \omega_{1}+a_{2} \omega_{2}+a_{3} \omega_{3}$. Ясно, что $c_{1}=3$. В силу теоремы $9 \mathcal{J}_{1}=\mathbf{N}_{S+1}^{o} \backslash\{1\}$ и $\mathbf{N}_{S+1}^{e} \backslash\{2\} \subset \mathcal{J}_{2}$.

Положим

$$
\begin{gathered}
\mu_{1}=\left\{\begin{array}{lll}
8 \omega_{1}+\left(a_{2}+a_{3}-6\right) \omega_{2} & \text { при } & a_{2}+a_{3} \geq 6 \text { и } a_{2}+a_{3} \neq 7 ; \\
8 \omega_{1} & \text { при } a_{2}+a_{3}=7 ; \\
4 \omega_{1}+3 \omega_{2} & \text { при } & a_{2}+a_{3}=5 ; \\
4 \omega_{1} & \text { при } & a_{2}+a_{3}=2,3 \text { или } 4 ;
\end{array}\right. \\
\mu_{2}=\left\{\begin{array}{lll}
5 \omega_{1}+\left(a_{2}+a_{3}-3\right) \omega_{2} & \text { при } a_{2}+a_{3} \geq 4 ; \\
3 \omega_{1}+2 \omega_{2} & \text { при } a_{2}+a_{3}=3 ; \\
3 \omega_{1}+\omega_{2} & \text { при } a_{2}+a_{3}=2 .
\end{array}\right.
\end{gathered}
$$

Во всех рассмотренных ситуациях $\mu_{1}, \mu_{2} \in \mathcal{S}(\omega)$. Определим модули $F_{1}$ и $F_{2}$, как в п. 3.2.3. Из теоремы 9 вытекает, что $1 \in J_{F_{1}}\left(x_{\mathbb{C}}\right)$ и $2 \in J_{F_{2}}\left(x_{\mathbb{C}}\right)$. Поэтому $J_{M}(x)=\mathbf{N}_{S+1}$.

Далее считаем, что $b_{1}>2$. 
3.2.5. $b_{1}=3$. В этом разделе $\omega=3 \omega_{1}+a_{2} \omega_{2}+a_{3} \omega_{3}$. Ясно, что $c_{1}=4$. Так как $b_{2}>1$, из теоремы 9 следует, что

$$
\begin{gathered}
\mathbf{N}_{S+1}^{o} \backslash\{1\} \subset \mathcal{J}_{2} \subset J_{M}(x), \\
\mathbf{N}_{S+1}^{e} \backslash\{2\} \subset \mathcal{J}_{1} \text { и } \mathcal{J}_{1}=\mathbf{N}_{S+1}^{e}, \quad \text { если } b_{2} \not \equiv 3 \quad(\bmod 6) .
\end{gathered}
$$

Пусть $b_{2} \equiv 3(\bmod 6)$. Тогда $b_{2} \geq 3$ и $\mu=5 \omega_{1}+\left(a_{2}+a_{3}-2\right) \omega_{2} \in \mathcal{S}(\omega)$. В силу теоремы 9

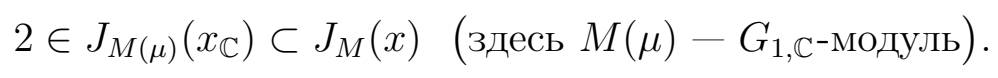

Значит, в любом случае $\mathbf{N}_{S+1}^{e} \subset J_{M}(x)$.

Поскольку $a_{1}=3$, то $\mathcal{S}(\omega)$ содержит вес $\nu=\left(a_{3}+i\right) \omega_{1}$ с $a_{3}+i \equiv$

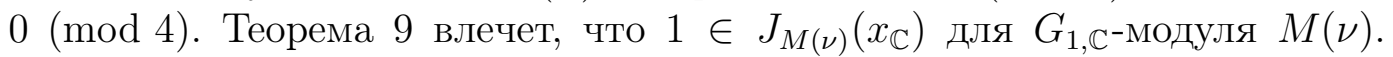
Следовательно, $J_{M}(x)=\mathbf{N}_{S+1}$.

3.2.6. Другие случаи. Пусть $b_{1}>3$ и один из весов $\mu_{1}$ или $\mu_{2}$ является исключительным. Так как $b_{2}>0$ и $c_{2}>0$, в силу теоремы 9 имеем

$$
\mathcal{J}_{1} \cup \mathcal{J}_{2}=\mathbf{N}_{S+1} \backslash\{1\}
$$

Далее строим вес $\nu=\left(a_{3}+i\right) \omega_{1} \in \mathcal{S}(\omega)$, как в п. 3.2.5, и убеждаемся, что $1 \in J_{M(\nu)}\left(x_{\mathbb{C}}\right)$. Следовательно, $J_{M}(x)=\mathbf{N}_{S+1}$.

При $n=3$ и $S<p$ теорема доказана.

3.3. $n>3$. В этом пункте предполагается, что $n>3$ и

$$
3 a_{1}+4 a_{2}+\cdots+4 a_{n}<p .
$$

Будем считать, что $\omega \neq a_{1} \omega_{1}$, так как этот случай рассмотрен в п. 3.1.

\section{Положим}

$H=G\left(\alpha_{1}, \alpha_{2}+\cdots+\alpha_{n-1}, \alpha_{n}\right), \quad \Gamma=G(n-2, n-1, n), \quad \lambda=\omega \mid H, \quad F=M(\lambda)$.

Ясно, что $H \cong C_{3}(K)$ и $\Gamma \cong C_{3}(K)$,

$$
\lambda=a_{1} \omega_{1}+\left(a_{2}+\cdots+a_{n-1}\right) \omega_{2}+a_{n} \omega_{3} .
$$

Так как $H$ и $\Gamma$ сопряжены, то $F \in \operatorname{Irr}(M \mid \Gamma)$. В силу следствия 4 и леммы $23 J_{F}(x) \subset J_{M}(x)$.

Множество $J_{F}(x)$ описано в п. 3.2. Ввиду доказанного в том пункте

$$
J_{F}(x)=J_{M}(x)=\mathbf{N}_{S+1},
$$

за исключением случаев, когда

$$
\lambda \in\left\{\omega_{2}, \omega_{3}, 2 \omega_{3}, 3 \omega_{3}, 5 \omega_{3}, \omega_{1}+\omega_{3}, 2 \omega_{1}+\omega_{3}, \omega_{2}+\omega_{3}, \omega_{1}+2 \omega_{3}, \omega_{1}+\omega_{2}+\omega_{3}\right\} .
$$


Далее рассмотрим эти случаи. Будем использовать анализ ограничения модуля $M$ на подсистемную подгруппу типа $C_{4}$. Положим

$$
\begin{gathered}
\Gamma_{1}=G(n-3, n-2, n-1, n), \\
\Gamma_{1, \mathbb{C}}=G_{\mathbb{C}}(n-3, n-2, n-1, n), \\
\Gamma_{\mathbb{C}}=G_{\mathbb{C}}(n-2, n-1, n), \quad \mu=\omega \mid \Gamma_{1} .
\end{gathered}
$$

В этом пункте $\delta$ и $\delta_{1}$ - максимальные короткие корни групп $\Gamma$ и $\Gamma_{1}$ соответственно, $M^{\prime}=M(\mu)$ - неприводимый $\Gamma_{1}$-модуль,

$$
\Sigma=\left\{\nu \mid M(\nu) \in \operatorname{Irr}\left(M_{\mathbb{C}}^{\prime} \mid \Gamma_{\mathbb{C}}\right)\right\}
$$

Существенно используется следующая

Лемма 24. Пусть $\left\langle\mu, \delta_{1}\right\rangle \leq p-7, \sigma \in \Sigma, Q=M(\sigma)-\Gamma$-модуль. Тогда

$$
J_{Q}(x) \subset J_{M}(x) .
$$

Доказательство. Покажем, что $Q \in \operatorname{Irr} M \mid$ Г. Поскольку корни $\delta$ и $\delta_{1}$ лежат в одной орбите относительно группы $W(\Gamma)$ и $\delta_{1} \in \Lambda^{+}\left(\Gamma_{1}\right)$, то $\langle\lambda, \delta\rangle \leq$ $\left\langle\mu, \delta_{1}\right\rangle \leq p-7$ при $\lambda \in \Lambda\left(M^{\prime}\right)$. Несложно проверить, что $\left\langle\rho_{\Gamma_{1}}, \delta_{1}\right\rangle=7$. Напомним, что $\left\langle\rho_{\Gamma}, \delta\right\rangle=5$. Поэтому $\left\langle\mu+\rho_{\Gamma_{1}}, \delta_{1}\right\rangle \leq p$ и $\left\langle\nu+\rho_{\Gamma}, \delta\right\rangle<p$, если $M(\nu) \in \operatorname{Irr}\left(M^{\prime} \mid \Gamma\right)$. Тогда в силу леммы 11 модуль $V(\mu)$ неприводим и модуль $V(\nu)$ неприводим, если $M(\nu) \in \operatorname{Irr}\left(M^{\prime} \mid \Gamma\right)$. Поэтому формальные характеры модулей $M^{\prime}$ и $M^{\prime} \mid \Gamma$ совпадают с формальными характерами модулей $M_{\mathbb{C}}^{\prime}$ и $M_{\mathbb{C}}^{\prime} \mid \Gamma_{\mathbb{C}}$ соответственно, а $\Sigma-$ с аналогичным множеством для $M^{\prime} \mid$ Г. Значит,

$$
Q \in \operatorname{Irr}\left(M^{\prime} \mid \Gamma\right) \subset \operatorname{Irr}(M \mid \Gamma)
$$

Остается воспользоваться следствием 4. Заметим, что в силу теоремы 3 $M^{\prime}$ - прямое слагаемое ограничения $M \mid \Gamma_{1}$, но для доказательства достаточно знать, что $M^{\prime}$ - композиционный фактор этого ограничения.

Обозначения леммы 24 используются до конца параграфа.

3.3.1. $\lambda=\omega_{2}$ или $\lambda=\omega_{3}$. Согласно доказанному в п. 3.2 имеем $J_{F}(x)=\{1,4,5\}$ или $J_{F}(x)=\{4,5\}$ соответственно. Ясно, что $\omega=\omega_{i}$ при $2 \leq i \leq n$.

Так как $\langle\omega, \gamma\rangle=1$, то очевидно, что $\operatorname{Irr}\left(M \mid \Gamma_{1}\right) \subset\left\{0, \omega_{1}, \omega_{2}\right\}$. Тогда из следствий 4 и 3 вытекает, что $J_{M}(x) \subset\{1,4,5\}$. Следовательно, $J_{M}(x)=$ $\{1,4,5\}$ при $\lambda=\omega_{2}$, т. е. при $\omega=\omega_{i}, 2 \leq i<n$.

Пусть $\omega=\omega_{n}$. Тогда $\mu=\omega_{4}$. Легко видеть, что $\left\langle\mu, \delta_{1}\right\rangle=2<p-7$. По теореме $8 \omega_{2} \in \Sigma$. Используем лемму 24 с $Q=M\left(\omega_{2}\right)$. Ввиду доказанного в п. 3.2 число 1 принадлежит $J_{Q}(x)$. Поэтому $J_{M}(x)=\{1,4,5\}$. 
3.3.2. $\lambda=2 \omega_{3}$. Здесь $\omega=2 \omega_{n}$. В силу теоремы $7 d_{\varphi}(x)=9$, и поэтому $J_{M}(x) \subset \mathbf{N}_{9}$.

Согласно доказанному в п. $3.2 J_{F}(x)=\mathbf{N}_{9} \backslash\{1,4\}$. Легко видеть, что $\mu=2 \omega_{4}$ и $\left\langle\mu, \delta_{1}\right\rangle=4 \leq p-7$. По теореме 8 имеем $2 \omega_{2} \in \Sigma$. Применим лемму 24 с $Q=M\left(2 \omega_{2}\right)$. Ввиду доказанного в п. $3.2\{1,4\} \subset J_{Q}(x) \subset J_{M}(x)$.

3.3.3. $\lambda=3 \omega_{3}$. В этом пункте $\omega=3 \omega_{n}$. Из теоремы 7 следует, что $J_{M}(x) \subset \mathbf{N}_{13}$. В силу доказанного в п. $3.2 J_{F}(x)=\mathbf{N}_{13} \backslash\{2\}$. Так как $S<p$, то $p \geq 13$. Легко видеть, что $\mu=3 \omega_{4}$ и $\left\langle\mu, \delta_{1}\right\rangle=6 \leq p-7$. Из теоремы 8 вытекает, что $3 \omega_{2} \in \Sigma$. Используем лемму 24 с $Q=M\left(3 \omega_{2}\right)$. Из доказанного в п. 3.2 следует, что $2 \in J_{Q}(x) \subset J_{M}(x)$.

3.3.4. $\lambda=5 \omega_{3}$. В этом пункте $\omega=5 \omega_{n}$. Так как $S<p$, то $p \geq 23$. В силу теоремы $7 J_{M}(x) \subset \mathbf{N}_{21}$. Из доказанного в п. 3.2 следует, что $J_{F}(x)=\mathbf{N}_{21} \backslash\{1\}$. Легко видеть, что $\mu=5 \omega_{4}$ и $\left\langle\mu, \delta_{1}\right\rangle=10<p-7$. Из теоремы 8 вытекает, что $5 \omega_{2} \in \Sigma$. Используем лемму 24 с $Q=M\left(5 \omega_{2}\right)$. Согласно доказанному в п. 3.2 заключаем, что $1 \in J_{Q}(x) \subset J_{M}(x)$.

3.3.5. $\lambda=\omega_{2}+\omega_{3}$. В этом пункте $\lambda=\omega_{2}+\omega_{3}$, т. е. $\omega=\omega_{i}+\omega_{n}$ при $2 \leq i \leq n-1$. В силу теоремы 7 и доказанного в п. $3.2 J_{M}(x) \subset \mathbf{N}_{9}$ и $J_{F}(x)=\mathbf{N}_{9} \backslash\{1\}$. Легко видеть, что $\mu \in\left\{\omega_{1}+\omega_{4}, \omega_{2}+\omega_{4}, \omega_{3}+\omega_{4}, \omega_{4}\right\}$. Нетрудно проверить, что во всех случаях $\left\langle\mu, \delta_{1}\right\rangle \leq 4 \leq p-7$. Положим

$$
\sigma= \begin{cases}\omega_{1}+\omega_{2} & \text { при } \quad \mu \in\left\{\omega_{1}+\omega_{4}, \omega_{2}+\omega_{4}, \omega_{3}+\omega_{4}\right\}, \\ \omega_{2} & \text { при } \quad \mu=\omega_{4}\end{cases}
$$

и $Q=M(\sigma)$. В силу теоремы $8 \sigma \in \Sigma$. Поэтому $J_{Q}(x) \subset J_{M}(x)$ ввиду леммы 24. С учетом доказанного в п. 3.2 имеем $1 \in J_{Q}(x)$.

3.3.6. $\lambda=\omega_{1}+\omega_{3}$. В этом пункте $\omega=\omega_{1}+\omega_{n}$. В силу теоремы 7 $J_{M}(x) \subset \mathbf{N}_{8}$. Из доказанного в п. 3.2 следует, что $J_{F}(x)=\mathbf{N}_{8} \backslash\{1\}$. Положим

$$
\Delta=G\left(\alpha_{1}, \alpha_{2}, \alpha_{3}+\cdots+\alpha_{n-1}, \alpha_{n}\right), \quad \tau=\omega \mid \Delta .
$$

Ясно, что $\Delta \cong C_{4}(K)$ и $\tau=\omega_{1}+\omega_{4}$. Так как подгруппы $\Gamma_{1}$ и $\Delta$ сопряжены в $G$, то $M \mid \Gamma_{1}$ имеет композиционный фактор $N$, изоморфный $M(\tau)$. Заметим, что $\left\langle\tau, \delta_{1}\right\rangle=3<p-7$. В силу леммы 11 модуль $V(\tau)$ неприводим. По теореме $8 N_{\mathbb{C}} \mid \Gamma_{\mathbb{C}}$ имеет композиционный фактор со старшим весом $\omega_{2}$. Пусть $Q^{\prime}=M\left(\omega_{2}\right)$ - неприводимый Г-модуль. Рассуждая, как в доказательстве леммы 24, можно установить, что $J_{Q^{\prime}}(x) \subset J_{M}(x)$. Ввиду доказанного в п. 3.2 заключаем, что $1 \in J_{Q^{\prime}}(x)$. Поэтому $J_{M}(x)=\mathbf{N}_{8}$.

3.3.7. $\lambda=2 \omega_{1}+\omega_{3}$. В этом пункте $\omega=2 \omega_{1}+\omega_{n}$. По теореме 7 $J_{M}(x) \subset \mathbf{N}_{11}$. Из доказанного в п. 3.2 вытекает, что $J_{F}(x)=\mathbf{N}_{11} \backslash\{1\}$. Далее повторяем рассуждения из п. 3.3.6, используя введенные там обозначения. Теперь $\tau=2 \omega_{1}+\omega_{4}$. Существенно, что $\left\langle\tau, \delta_{1}\right\rangle=4 \leq p-7$. 
3.3.8. $\lambda=\omega_{1}+2 \omega_{3}$. В этом пункте $\lambda=\omega_{1}+2 \omega_{3}$, т. е. $\omega=\omega_{1}+2 \omega_{n}$. В силу теоремы 7 и доказанного в п. $3.2 J_{M}(x) \subset \mathbf{N}_{12}$ и $J_{F}(x)=\mathbf{N}_{12} \backslash\{1\}$. Далее рассуждаем, как в п. 3.3.6, выбрав другую подгруппу $\Delta$ типа $C_{4}$. Сейчас мы положим $\Delta=G\left(1,2,3,2 \varepsilon_{4}\right)$ и $\tau=\omega \mid \Delta$. Ясно, что $\Delta \cong C_{4}(K)$ и $\tau=\omega_{1}+2 \omega_{4}$. Поскольку подгруппы $\Delta$ и $\Gamma_{1}$ сопряжены в $G$, то $M \mid \Gamma_{1}$ имеет композиционный фактор $N$, изоморфный $M(\tau)$. Заметим, что $p \geq 13$, ибо $S<p ;\left\langle\tau, \delta_{1}\right\rangle=5<p-7$; и ввиду теоремы $8 N_{\mathbb{C}} \mid \Gamma_{\mathbb{C}}$ имеет композиционный фактор со старшим весом $2 \omega_{2}$. Пусть $Q^{\prime} \cong M\left(2 \omega_{2}\right)-\Gamma$-модуль. Используя аргументы из доказательства леммы 24 и п. 3.3.6, можно установить, что $J_{Q^{\prime}}(x) \subset J_{M}(x)$. В силу доказанного в п. 3.2 получаем, что $1 \in J_{Q^{\prime}}(x)$.

3.3.9. $\lambda=\omega_{1}+\omega_{2}+\omega_{3}$. В этом пункте $\lambda=\omega_{1}+\omega_{2}+\omega_{3}$, т. е. $\omega=$ $\omega_{1}+\omega_{i}+\omega_{n}$ с $2 \leq i \leq n-1$. Ввиду теоремы 7 и доказанного в п. 3.2 $J_{M}(x) \subset \mathbf{N}_{12}$ и $J_{F}(x)=\mathbf{N}_{12} \backslash\{1\}$.

Далее рассуждаем, как в пп. 3.3.6-3.3.8. Положим

$$
\Delta=G\left(\alpha_{1}, \alpha_{2}+\cdots+\alpha_{n-2}, \alpha_{n-1}, \alpha_{n}\right) \text { и } \tau=\omega \mid \Delta .
$$

Ясно, что $\Delta \cong C_{4}(K)$ и

$$
\tau=\left\{\begin{array}{lll}
\omega_{1}+\omega_{2}+\omega_{4} & \text { при } & i<n-1 \\
\omega_{1}+\omega_{3}+\omega_{4} & \text { при } & i=n-1 .
\end{array}\right.
$$

Учитывая, что подгруппы $\Gamma_{1}$ и $\Delta$ сопряжены в $G$, и используя теорему 8 и рассуждения из доказательства леммы 24 и п. 3.3.6, устанавливаем, что $M \mid \Gamma_{1}$ имеет композиционный фактор $F$, изоморфный $M(\tau), F_{\mathbb{C}} \mid \Gamma_{\mathbb{C}}$ имеет композиционный фактор со старшим весом $2 \omega_{2}$ и что для Г-модуля $Z=M\left(2 \omega_{2}\right)$ множество $J_{Z}(x)$ содержится в $J_{M}(x)$. Из доказанного в п. 3.2 следует, что $1 \in J_{Z}(x)$. При этом существенно, что $p \geq 13$ и $\left\langle\tau, \delta_{1}\right\rangle=5<$ $p-7$.

Итак, при $S<p$ все возможности рассмотрены и теорема 1 доказана.

Напомним, что все конечномерные рациональные модули для полупростых алгебраических групп в характеристике 0 вполне приводимы (см. [19, теорема 6.3]).

Легко видеть, что рассуждения этого параграфа можно модифицировать для характеристики 0. Это позволяет доказать теорему 2.

\section{Список литературы}

1. Бурбаки Н. Группы и алгебры Ли. Гл. IV-VI. М.: Мир, 1972.

2. Бурбаки Н. Группы и алгебры Ли. Гл. VII-VIII. М.: Мир, 1978.

3. Бусел Т. С., Супруненко И. Д. Блочная структура образов регулярных унипотентных элементов из подсистемных подгрупп типа $C_{2}$ в непри- 
водимых представлениях групп типа $C_{n}$ с локально малыми старшими весами // Докл. НАН Беларуси. 2016. Т. 60, № 1. С. 12-17.

4. Величко М. В. О поведении корневых элементов в модулярных представлениях симплектических групп // Тр. Ин-та матем. 2006. Т. 14, № 2. C. 28-34.

5. Величко М. В., Супруненко И. Д. Малые квадратичные элементы в представлениях специальной линейной группы с большими старшими весами // Зап. научн. сем. ПОМИ. 2007. Т. 343. С. 84-120.

6. Величко М. В. Структура блоков Жордана образов длинных корневых элементов в модулярных представлениях групп типов $B_{n}$ и $F_{4} / /$ Тр. Ин-та матем. 2011. Т. 19, № 2. С. 7-11.

7. Желобенко Д. П. Классические группы. Спектральный анализ конечномерных представлений // УМН. 1962. Т. 17, № 1(103). С. 27-120.

8. Залесский A. Е., Супруненко И. Д. Срезанные симметрические степени естественных реализаций групп $S L_{m}(P)$ и $S p_{m}(P)$ и их ограничения на подгруппы // Сиб. матем. журн. 1990. Т. 31, № 4. С. 33-46.

9. Осиновская А. А. Регулярные унипотентные элементы из подсистемных подгрупп типа $C_{2}$ в представлениях // Tp. Ин-та матем. 2009. T. 17, № 1. С. 119-126.

10. Осиновская А. А., Супруненко И. Д. Унипотентные элементы из подсистемных подгрупп типа $A_{3}$ в представлениях специальной линейной группы // Докл. НАН Беларуси. 2012. Т. 56, № 4. С. 11-15.

11. Премет A. А. Веса инфинитезимально неприводимых представлений групп Шевалле над полем простой характеристики // Maтем. сб. 1987. T. 133(175), № 2(6). С. 167-183.

12. Стейнберг Р. Лекиии о группах Шевалле. М.: Мир, 1975.

13. Супруненко И. Д. Минимальные полиномы элементов порядка $p$ в неприводимых представлениях групп Шевалле над полями характеристики $p / /$ Тр. Ин-та математики СО РАН. Новосибирск: Изд-во Ин-та математики, 1996. Т. 30: Вопросы алгебры и логики. С. 126-163.

14. Фейт У. Теория представлений конечных групп. М.: Наука, 1990.

15. Borel A. Properties and linear representations of Chevalley groups // Semin. Algebr. Groups Related Finite Groups (Princeton 1968/69) / Lecture Notes in Math.; N 131. Berlin: Springer, 1970. P. A1-A55.

16. Braden B. Restricted representations of classical Lie algebras of type $A_{2}$ and $B_{2} / /$ Bull. Amer. Math. Soc. (N.S.). 1967. V.73. P.482-486.

17. Carter R. W. Finite Groups of Lie Type: Conjugacy Classes and Complex Characters. New York etc.: John Wiley and Sons, 1985. 
18. Donkin S. On tilting modules for algebraic groups // Math. Z. 1993. V.212, N 1. P. 39-60.

19. Humphreys J. Modular Representations of Finite Groups of Lie Types / London Math. Soc. Lecture Note Ser. Cambridge: Cambridge Univ. Press, 2006. V. 326.

20. Jantzen J. C. Representations of Algebraic Groups. 2nd ed. / Math. Surveys and Monographs. Providence, RI: Amer. Math. Soc., 2003. V. 107.

21. Osinovskaya A. A. Nilpotent Elements in Irreducible Representations of Simple Lie Algebras of Small Rank / Preprint: V. 554, N 5. Minsk: National Academy of Sciences of Belarus, Institute of Mathematics, 1999.

22. Osinovskaya A. A. Restrictions of representations of algebraic groups of types $E_{n}$ and $F_{4}$ to naturally embedded $A_{1}$-subgroups and the behavior of root elements // Comm. Algebra. 2005. V.33, N 1. P. 213-220.

23. Osinovskaya A. A. and Suprunenko I. D. On the Jordan block structure of images of some unipotent elements in modular irreducible representations of the classical algebraic groups // J. Algebra. 2004. V. 273, N 2. P. 586-600.

24. Seitz G. M. The Maximal Subgroups of Classical Algebraic Groups / Mem. Amer. Math. Soc. 1987. V.67, N 365.

25. Seitz G. M. Unipotent elements, tilting modules, and saturation // Invent. Math. 2000. V.141, N 3. P. 467-502.

26. Smith S. D. Irreducible modules and parabolic subgroups // J. Algebra. 1982. V. 75. P. 286-289.

27. Suprunenko I. D. The Minimal Polynomials of Unipotent Elements in Irreducible Representations of the Classical Groups in Odd Characteristic / Mem. Amer. Math. Soc. 2009. V. 200, N 939.

28. Velichko M. V. On the behavior of the root elements in irreducible representations of simple algebraic groups // Тр. Ин-та матем. 2005. Т. 13, N 2. C. $116-121$.

Бусел Татьяна Сергеевна

Супруненко Ирина Дмитриевна

Институт математики НАН Беларуси, ул. Сурганова, 11,

Минск, 220072 БЕЛАРУСЬ.

E-mail: tbusel@im.bas-net.by;

E-mail: suprunenko@im.bas-net.by
Поступила в редакцию 10 января 2018 г.

Получена после доработки 3 октября 2018 г.

Принята к публикации 10 октября 2018 г. 